\title{
Efficient Cryptanalysis of Homophonic Substitution Ciphers
}

\author{
Amrapali Dhavare* Richard M. Low ${ }^{\dagger}$ Mark Stamp ${ }^{\ddagger}$
}

\begin{abstract}
Substitution ciphers are among the earliest methods of encryption. Examples of classic substitution ciphers include the well-known simple substitution and the less well-known homophonic substitution. Simple substitution ciphers are indeed simpleboth in terms of their use and their cryptanalysis. Homophonic substitutions are also easy to use, but far more challenging to break. Even with modern computing technology, homophonic substitutions can present a significant cryptanalytic challenge.

This paper focuses on the design and implementation of an efficient algorithm to break homophonic substitution ciphers. We employ a nested hill climb approach that generalizes the fastest known attack on simple substitution ciphers. We test our algorithm on a wide variety of homophonic substitutions and provide success rates as a function of both the ciphertext alphabet size and ciphertext length. Finally, we apply our technique to the "Zodiac 340" cipher, which is an unsolved message created by the infamous Zodiac killer.
\end{abstract}

Keywords: homophonic substitution cipher, simple substitution cipher, hill climb, heuristic search, Zodiac 340 cipher

\section{Introduction}

Substitution ciphers are among the oldest encryption methods - they are simple, intuitive, and widely studied [21]. Many variants of the substitution cipher have been developed, including monoalphabetic systems, which employ a fixed substitution, and polyalphabetic systems, where the substitution varies. Example of monoalphabetic substitutions include the simple substitution and homophonic substitution ciphers. Examples of polyalphabetic substitutions include the Vigenére cipher and World War II era rotor cipher machines, such as the Enigma. Here, we are concerned with monoalphabetic substitutions.

${ }^{*}$ Department of Computer Science, San Jose State University

$\dagger$ Department of Mathematics, San Jose State University

${ }_{\ddagger}^{\ddagger}$ Department of Computer Science, San Jose State University: stamp@cs.sjsu.edu 
For the simple substitution cipher, the plaintext to ciphertext mapping is one-toone. In contrast, homophonic substitution ciphers are many-to-one, that is, multiple ciphertext symbols can map to one plaintext symbol.

The simple substitution cipher is indeed simple in terms of its use, but it is vulnerable to elementary statistical analysis. For example, the most common ciphertext symbol corresponds to the most common plaintext symbol, which for English plaintext is most likely the letter E. For a homophonic substitution, the plaintext statistics tend to be flattened, since multiple ciphertext symbols can correspond to a single plaintext symbol. For example, if the plaintext is English and the letter E corresponds to multiple symbols, none of those symbols may stand out as having an unusually high frequency. There are several fast and effective attacks on the simple substitution, but homophonic substitutions are inherently more challenging.

Our motivation for considering homophonic substitution ciphers is the unsolved "Zodiac 340," which was created by the infamous Zodiac killer in 1969 [5]. Another Zodiac cipher, the "Zodiac 408," was a homophonic substitution and it was broken within days of its publication [5]. In contrast, the Zodiac 340 has so far proved resistant to cryptanalysis. We have more to say about these ciphers in Section 6 .

In this paper, we discuss the design and implementation of an efficient, general attack on homophonic substitution ciphers. We also provide extensive test results. The attack proposed here can be viewed as a generalization of the fastest known algorithm for breaking simple substitutions [7]. However, a direct generalization of the algorithm in [7] is not sufficient to solve a homophonic substitution. Therefore, we combine our generalized simple substitution algorithm with an additional hill climb layer. As with the simple substitution algorithm, only digram frequencies are used for scoring.

This paper is organized as follows. Section 2 briefly covers relevant background information. Section 3 describes a fast algorithm for solving simple substitutions [7], which we generalize to homophonic substitutions in Section 4. Then in Section 5, we present results for some of the many tests we have conducted. Section 6 covers the Zodiac ciphers and a related challenge problem, and we give the results of our attack when applied to these ciphers. Finally, Section 7 contains our conclusion and suggestions for future work.

\section{Background}

In this section, we consider simple substitution and homophonic substitution ciphers. We also discuss letter frequencies and the role they can play in the cryptanalysis of these substitution ciphers. Finally, we conclude this section with a discussion of hill climbing in the context of substitution cipher cryptanalysis. 


\subsection{Simple Substitution}

Substitution ciphers can be defined as ciphers in which every plaintext symbol has a ciphertext symbol substituted for it, and the original position of the plaintext symbol is retained in the ciphertext [10]. There are various ways in which the substitutions can be done. In this section we discuss the simplest of the substitution ciphers which, appropriately, is known as a simple substitution.

A simple substitution is a one-to-one mapping, in the sense that each plaintext symbol corresponds to one ciphertext symbol. This makes encryption and decryption straightforward, but it is well-known that the simple substitution is vulnerable to elementary statistical analysis [21]. In particular, frequency analysis can be used to attack the simple substitution.

An example of a simple substitution key is given in Table 1. In this particular example, each plaintext letter is replaced with the letter that is 13 positions ahead in the alphabet but, in general, any permutation of the alphabet can serve as a key.

Table 1: Simple Substitution Key

\begin{tabular}{l|lll}
\hline plaintext & A B C D E F G H I J K L M N O P Q R S T U V W X Y Z
\end{tabular}
ciphertext N O P Q R S T U V W X Y Z A B C D E F G H I J K L M

Using the key in Table 1, the plaintext HELLO encrypts to ciphertext URYYB. For a simple substitution, any given plaintext letter always encrypts to the same ciphertext letter and, consequently, each ciphertext letter has the same frequency in ciphertext as its corresponding plaintext letter has in the plaintext. So, given the five-letter ciphertext URYYB, a cryptanalyst knows that the third and fourth plaintext letters are identical.

Suppose that the plaintext is English and only the 26 letters appear, i.e., there is no word-space, punctuation, case, etc. Then the simple substitution key space consists of the $26 ! \approx 2^{88}$ possible permutations of the alphabet. Consequently, the expected work for an exhaustive search is about $2^{87}$. Supposing that we can test one million keys per second, an exhaustive search will take about $2^{87} / 10^{6}=1.55 \times 10^{20}$ seconds, or about $4.9 \times 10^{12}$ years. Therefore, under any reasonable assumptions, an exhaustive key search is infeasible.

In spite of the enormous work factor for an exhaustive search, breaking a simple substitution cipher is an elementary textbook exercise in cryptanalysis. Assuming English plaintext and a message of reasonable length, the highest-frequency ciphertext letter most likely corresponds to plaintext $\mathrm{E}$, since $\mathrm{E}$ is the most common letter in English. This trivial observation can be extended to an effective attack by comparing English letter frequencies to ciphertext letter frequencies. Once we have determined a few of the high-frequency letters, common letter patterns become apparent and the key can then be completely recovered. 
The following is a general outline for a systematic approach to attacking a simple substitution.

1. Let $\mathrm{score}=\infty$

2. Construct an initial putativeKey

3. Parse the ciphertext using putativeKey

4. Compute newScore based on the resulting putative plaintext

5 . If newScore $<$ score then let key $=$ putativeKey and score $=$ newScore

6. Modify key to obtain a new putativeKey

7. Goto 3

Here, we are assuming that score $\geq 0$, with score $=0$ representing a perfect match, and the bigger the score, the worse the result. Also, a suitable stopping criteria is needed, such as the score falling below a given threshold or a maximum number of iterations exceeded. Note that to implement an attack based on this outline, we must have practical answers to the following questions.

- How can we determine an initial putative key?

- How can we systematically modify the key?

- Given a putative key, how can we compute a score?

A reasonable guess for the initial putative key can be obtained by sorting the ciphertext letters by frequency and matching the letters with the corresponding letter frequencies of English. That is, the most frequent ciphertext letter would be mapped to plaintext $\mathrm{E}$, the second most frequent would be mapped to $\mathrm{T}$, and so on.

To modify the key, we can simply swap pairs of letters in the putative key. Since any permutation can be obtained from another permutation by repeated swapping, this elementary approach enables us to, in principle, obtain any key.

To compute a score, we could look for dictionary words [12], but given a sufficiently long ciphertext, an alternative approach is to use English digram frequencies (and, possibly, trigrams and higher-order-grams). We discuss English letter frequencies and English digram statistics in more detail below.

The major drawback to a straightforward attack on the simple substitution is that the ciphertext must be re-parsed at every iteration. That is, for each modification to the putative key we must recompute the putative plaintext. Consequently, the work can become prohibitively expensive as the length of the ciphertext increases. We discuss a more efficient approach in Section 3. This fast algorithm virtually eliminates any dependence on the length of the ciphertext, and it provides an efficient letter-swapping scheme.

Next, we discuss homophonic substitution ciphers. In general, homophonic substitutions are much more resistant to attacks based on frequency analysis as compared to simple substitutions. 


\subsection{Homophonic Substitution}

Instead of using a one-to-one mapping as in a simple substitution, a homophonic substitution employs a one-to-many mapping. Consequently, there may be more than one possible substitution for a given plaintext letter. However, each ciphertext symbol can represent only one plaintext letter, so the decryption is unique. Such a mapping tends to flatten the frequency statistics in the resulting ciphertext and consequently makes attacks based on frequency analysis more difficult.

An example of a homophonic substitution cipher is given in Table 2, where we have used some non-alphabetic symbols, since we require more than 26 ciphertext symbols. For the key in Table 2, any of the symbols R, 3, or 9 can be substituted for a plaintext E, and either Y or 6 can be substituted for plaintext L. So, using this key, plaintext HELLO can be encrypted as U96YB. In this case, a cryptanalyst has no indication that ciphertext 6 and $Y$ both represent the same plaintext letter.

Table 2: Homophonic Substitution Key

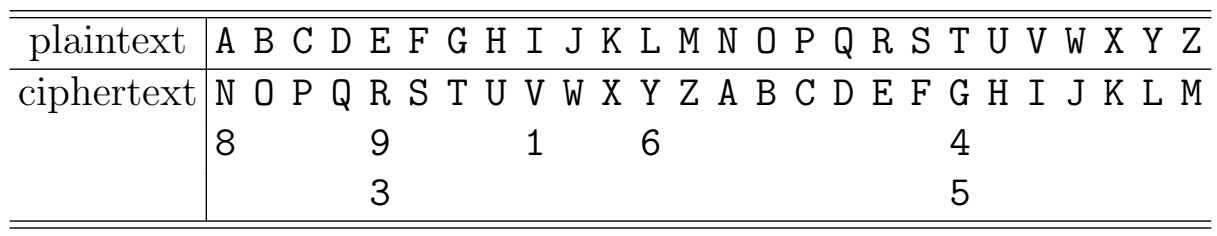

Suppose that a given homophonic substitution cipher has $n$ distinct ciphertext symbols and the plaintext is English. Assuming that each of the 26 plaintext letters maps to at least one ciphertext symbol, the theoretical key space is of size

$$
\left(\begin{array}{c}
n \\
26
\end{array}\right) 26 ! 26^{n-26}<26^{n} \approx 2^{4.7 n}
$$

Recall that for the simple substitution, the key space is of size $26 ! \approx 2^{88}$, with an exhaustive search having a work factor of $2^{87}$, on average.

Using the upper bound of $2^{4.7 n}$, and assuming that we can test a million keys per second, an exhaustive search on a homphonic substitution with $n=100$ ciphertext symbols will require about $2^{470} / 2^{20}=2^{450}$ seconds, or $9.2 \times 10^{127}$ years. For a cipher with 62 symbols - such as the Zodiac 340 cipher-the same assumptions give an exhaustive key search time of "only" $1.59 \times 10^{74}$ years. As discussed in the previous section, under the same assumptions, an exhaustive search on a simple substitution requires a paltry $4.9 \times 10^{12}$ years.

The point here is that an exhaustive search is out of the question. In Sections 3 and 4 we consider efficient attacks on simple substitutions and homophonic substitutions, respectively. These attacks rely on statistical information that leaks from the plaintext into the ciphertext. 


\subsection{Digram Frequencies}

Expected English letter frequencies are given in Figure 1. The most frequent letter in English is E, accounting for nearly 13\% of all letters. The least frequent English letters are $\mathrm{Q}$ and $\mathrm{J}$, each of which has an expected frequency that is much less than $1 \%$. In contrast, for a random collection of letters, each of the 26 letters would have an expected frequency of $1 / 26 \approx 3.85 \%$.

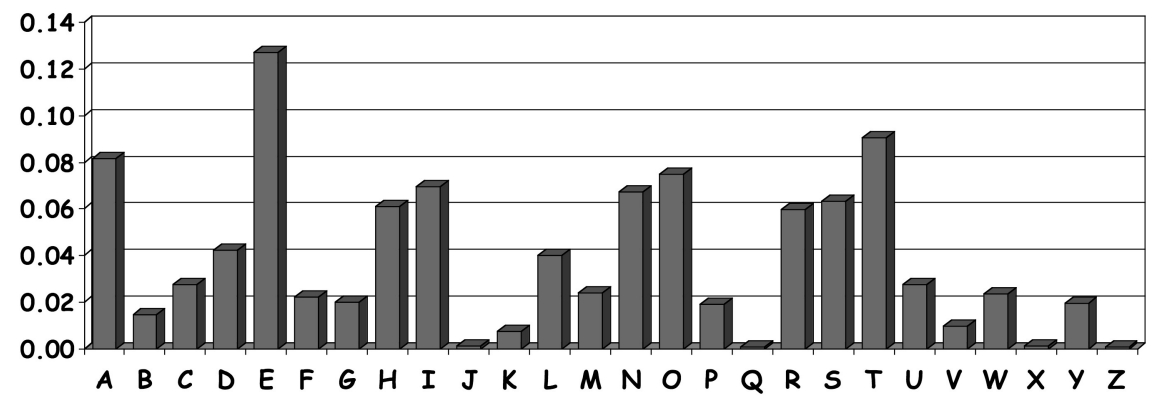

Figure 1: English Letter Frequencies

Digrams are consecutive pairs of symbol. For English, there are $26^{2}$ digrams and their expected frequencies vary widely. For example, the most common English digram is $\mathrm{TH}$, which accounts for about $1.5 \%$ of all digrams, while there are many digrams that never occur, such as QJ. For a random collection of letters, the expected frequency of each digram would be $1 / 26^{2} \approx 0.15 \%$.

Table 3 contains English digram frequencies, where the cells are color-coded to indicate the frequency - red represents higher frequencies while blue represents lower frequencies [4]. Note that Table 3 includes the symbols "^" and "\$" which indicate the beginning and ending of words, respectively. If we include word boundaries, the most common digram is $\mathbf{S} \$$, that is, the letter $\mathbf{S}$ at the end of a word.

Jakobsen [7] gives a fast algorithm for breaking a simple substitution, using only English digram frequencies. By searching for dictionary words (or trigrams and, possibly, higher order "grams"), we may be able to reduce the ciphertext length requirements. However, by focusing our attention on digram frequencies, it is possible to design an extremely efficient attack on the simple substitution. Specifically, we are able to avoid re-parsing the ciphertext for each change in the putative key.

We discuss Jakobsen's simple substitution algorithm in Section 3. Our generalization of this attack to the homophonic substitution is covered in Section 4. But first, we briefly discuss heuristic methods, with an emphasis on hill climbing.

\subsection{Hill Climbing}

As noted above, the homophonic substitution cipher has a keyspace that is far too large for an exhaustive search. Therefore, we consider heuristic search techniques. 
Table 3: English Digram Frequencies [4]

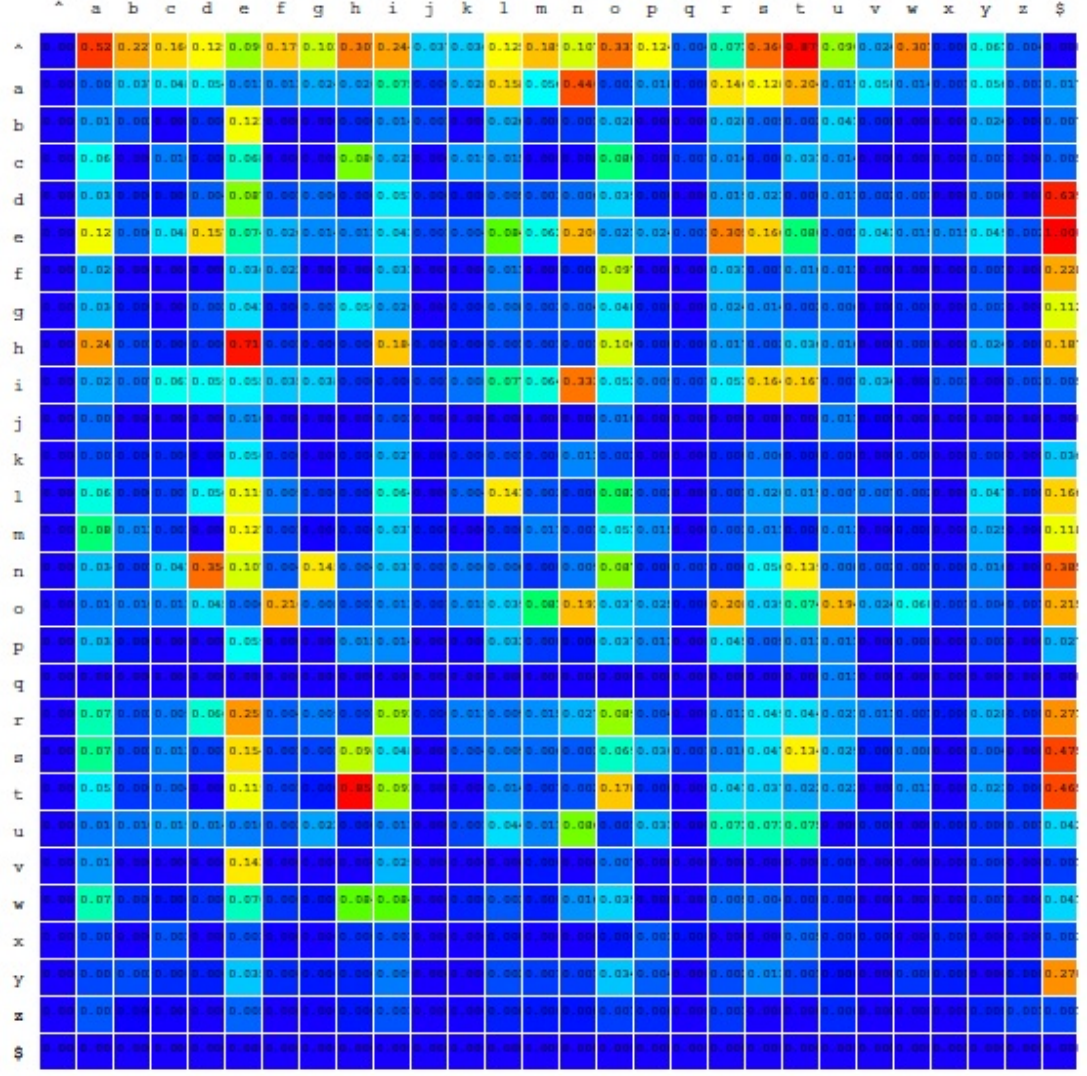

According to [8] (italics appear in the original),

The term heuristic algorithm is used to describe an algorithm (usually a randomized algorithm) that tries to find a certain combinatorial structure or solve an optimization problem by the use of heuristics. The Oxford Reference Dictionary defines heuristic as "serving or helping to find out or discover; proceeding by trial and error". In the context of a heuristic algorithm, a heuristic will be a method of performing a minor modification, or a sequence of modifications, of a given solution or partial solution in order to obtain a different solution or partial solution.

In other words, a heuristic is an "educated guess" and a heuristic search uses a series of such guesses in an attempt to find a reasonable solution to a given combinatorial problem.

The obvious advantage of a heuristic search is that it can be much faster than a brute force approach. However, there is no guarantee that a heuristic algorithm 
will find an optimal solution, or even a reasonable approximation [15]. But for most substitution ciphers, an exhaustive search is out of the question, so it is natural to consider heuristic techniques.

Hill climbing is an an iterative technique that can be viewed as a type of heuristic search. During each iteration of the hill climb, a small modification is made to the putative solution, which is then evaluated to determine whether it is an improvement over the previous solution. If the modified solution is better, then the modified solution becomes the new putative solution; otherwise we make no change to the putative solution. Thus, with every iteration, the solution either improves or stays the same. That is, the algorithm can only climb "up" towards a better solution - it can never produce a worse solution.

To design any hill climb algorithm, two issues need to be addressed. First, we need a way to incrementally modify a given solution during each iteration. Second, we need a scoring function to quantify the "goodness" of a given putative solution.

A hill climb algorithm can find locally optimal solutions but, as with any heuristic technique, there is no assurance that it will find a globally optimal solution. Hill climb algorithms tend to be very sensitive to the initial guess, as illustrated in Figure 2. If the initial guess is sufficiently close to a globally optimal solution, then we will climb to that optimal solution. In general, the hill climb will find a nearby local maximum which, depending on the geometry of the parameter space, might not be a useful solution to the problem. Many heuristic search techniques try to overcome this limitation by occasionally choosing inferior solutions, which makes it possible to jump from one "hill" to another. For example, in genetic algorithms, a mutation factor is used for this purpose. With a straightforward hill climb algorithm, multiple initial guesses will increase our chance of finding a satisfactory solution, since we can then choose the best of the resulting local maxima.

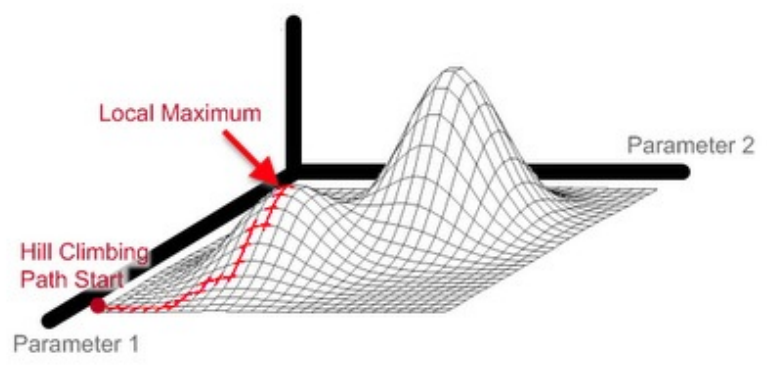

Figure 2: Hill Climbing [9]

Hill climbing works well on many substitution ciphers, including some polyalphabetic cipher machines, such as the World War II-era Japanese Purple cipher [20]. For substitution ciphers, the closer a putative key is to the actual key, the closer the corresponding putative plaintext is to the actual plaintext. This is the crucial 
feature that enables a hill climb (or other heuristic search) to succeed. In contrast, for a modern cipher, any putative key that differs from the actual key should yield putative plaintext that is uncorrelated to the actual plaintext. In other words, if just one bit differs from the actual key, the resulting putative plaintext is no closer to the actual plaintext than for a random key.

Figure 3 shows the results of an experiment conducted on the Advanced Encryption Standard (AES), a modern block cipher [2]. In the graph, the $x$ axis represents the closeness of the putative key to the actual key, ranging from $55 \%$ to $100 \%$. The $y$ axis in Figure 3 represents the percentage of similarity between the putative plaintext and the actual plaintext. The graph shows that as the putative key gets closer to the actual key, the similarity between the putative plaintext and the actual plaintext remains essentially random (at random, we expect about half of the bits to match). Only when the putative key is exactly equal to the actual key does the putative plaintext match the actual plaintext to a degree that is significantly better than random. In other words, the search space is essentially flat, with the exception of a spike at the correct key value (i.e., a delta function), so there are no meaningful hills to climb.

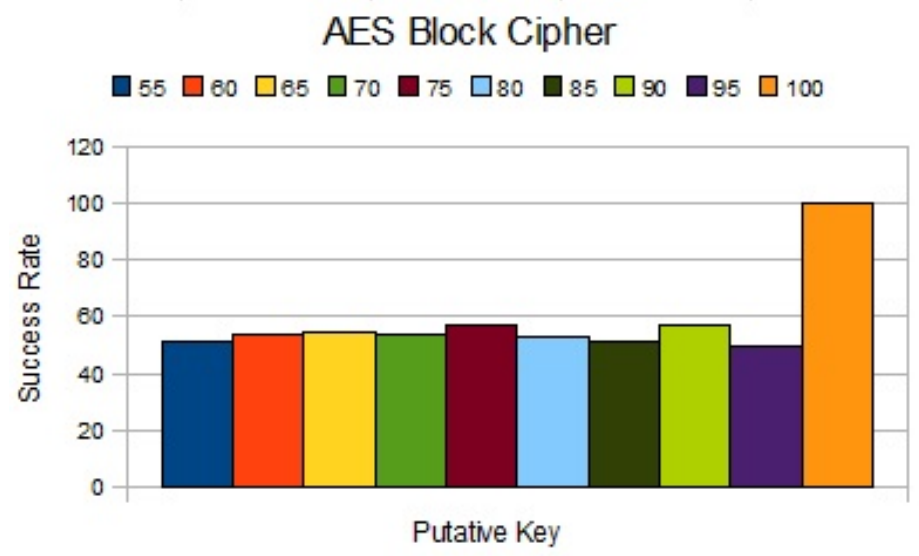

Figure 3: AES Block Cipher Success Rate

\section{Fast Attack on Simple Substitution}

In this section, we discuss Jakobsen's fast algorithm for breaking a simple substitution cipher [7]. Throughout this section we assume that the plaintext language is English, and the 26 letters of the alphabet are also used as the ciphertext symbols. However, the same principles apply to other languages and, of course, we can use arbitrary symbols to represent the ciphertext.

Jakobsen's algorithm uses English digram statistics for scoring and the ciphertext is only parsed once to construct an initial digram distribution matrix. All subsequent 
scoring is done by directly manipulating the plaintext digram distribution matrix and then comparing the result to the expected English digram distribution matrix.

As mentioned in Section 2.1, to fully specify a simple substitution attack, we need to answer the following questions:

- How can we determine an initial putative key?

- How can we systematically modify the key?

- Given a putative key, how can we compute a score? That is, we need a way to quantify the "goodness" of a putative key.

For this fast attack, we choose the initial key that best matches with English monograph statistics. That is, we assume the most frequent ciphertext letter maps to plaintext $\mathrm{E}$, the second most frequent maps to $\mathrm{T}$, and so on.

We modify the putative key by swapping elements, as follows. First, denote the putative key as $K=\left(k_{1}, k_{2}, \ldots, k_{26}\right)$, where $K$ is a permutation of the 26 English letters. The swapping will be done systematically through a series of rounds. In the first round, all adjacent elements are selected for swapping, that is, $k_{1}$ is swapped with $k_{2}$, then $k_{2}$ is swapped with $k_{3}$, then $k_{3}$ with $k_{4}$, and so on. In the second round, the elements in $K$ at distance two are swapped, while in the third round, elements at a distance of three are swapped, and so on. Let "| represent the swapping operation. Then the following series of swaps are performed:

$$
\begin{aligned}
& \text { round 1: } \quad k_{1}\left|k_{2} \quad k_{2}\right| k_{3} \quad k_{3}\left|k_{4} \quad \ldots \quad k_{23}\right| k_{24} \quad k_{24}\left|k_{25} \quad k_{25}\right| k_{26} \\
& \text { round 2: } \quad k_{1}\left|k_{3} \quad k_{2}\right| k_{4} \quad k_{3}\left|k_{5} \quad \ldots \quad k_{23}\right| k_{25} \quad k_{24} \mid k_{26} \\
& \text { round 3: } \quad k_{1}\left|k_{4} \quad k_{2}\right| k_{5} \quad k_{3}\left|k_{6} \quad \ldots \quad k_{23}\right| k_{26} \\
& \begin{array}{lll}
\vdots & \vdots
\end{array} \\
& \text { round 24: } \quad k_{1}\left|k_{24} \quad k_{2}\right| k_{25} \quad k_{3} \mid k_{26} \\
& \text { round 25: } k_{1}\left|k_{25} \quad k_{2}\right| k_{26} \\
& \text { round } 26: \quad k_{1} \mid k_{26}
\end{aligned}
$$

Note that all $\left(\begin{array}{c}26 \\ 2\end{array}\right)$ pairs of putative key elements are swapped once.

The third crucial point is that we must have an efficient method for scoring a putative key. This is accomplished by computing a score that measures the closeness of the putative plaintext digram matrix to the expected English language digram matrix. For two matrices $X=\left\{x_{i j}\right\}$ and $Y=\left\{y_{i j}\right\}$ with the same dimensions, let

$$
d(X, Y)=\sum_{i, j}\left|x_{i j}-y_{i j}\right| .
$$

We use this distance function to define a score as follows. Let $D=\left\{d_{i j}\right\}$ be the digram frequency matrix for the putative plaintext corresponding to a putative key $K$ and let $E=\left\{e_{i j}\right\}$ be the expected digram frequency matrix for English. Then the score 
for the putative key $K$ is defined as

$$
\operatorname{score}(K)=d(D, E)=\sum_{i, j}\left|d_{i j}-e_{i j}\right|
$$

Note that score $\geq 0$, with score $=0$ being a perfect match, and the smaller the score, the better the key $K$.

The efficiency of Jakobsen's algorithm derives from the fact that when we alter the putative key by swapping elements, we can simply modify the $D$ matrix, without re-parsing the ciphertext. When key elements are swapped, we only need to modify digram frequencies that begin or end with the swapped elements - all other digrams are unaffected. It is easy to show that swapping elements of the putative key has the effect of swapping the corresponding rows and columns of the $D$ matrix, leaving the remainder of the $D$ matrix unchanged.

To illustrate Jakobsen's algorithm, we consider a simple substitution example on the restricted 8-letter alphabet

$$
\mathrm{E}, \mathrm{T}, \mathrm{I}, \mathrm{S}, \mathrm{H}, \mathrm{R}, \mathrm{L} \text {, and } \mathrm{K} \text {. }
$$

Note that these letters are listed in descending order of their expected frequencies in English. Based on this limited alphabet, suppose we are given the simple substitution ciphertext

$$
\text { RIHILKHIERSKILEKKLKTRSKHRIHILKHLREIRTRSKLKTRSKHHLEKRS. }
$$

For the ciphertext in (3), the frequency counts are

$$
\begin{array}{cccccccc}
\mathrm{E} & \mathrm{T} & \mathrm{I} & \mathrm{S} & \mathrm{H} & \mathrm{R} & \mathrm{L} & \mathrm{K} \\
4 & 3 & 7 & 5 & 7 & 9 & 7 & 11
\end{array}
$$

Consequently, our initial guess for the key is

$$
\begin{array}{rllllllll}
\text { Plaintext: } & \text { E } & \text { T } & \text { I } & \text { S } & \text { H } & \text { R } & \text { L } & \text { K } \\
\text { Ciphertext: } & \text { K } & \text { R } & \text { I } & \text { L } & \text { H } & \text { S } & \text { E } & \text { T }
\end{array}
$$

Using this initial putative key, we find the initial putative plaintext corresponding to the ciphertext in (3) is

TIHISEHILTREISLEESEKTREHTIHISEHSTLITKTRESEKTREHHSLETR 
For this initial plaintext, the digram frequency matrix is

\begin{tabular}{c|cccccccc} 
& $\mathrm{E}$ & $\mathrm{T}$ & $\mathrm{I}$ & $\mathrm{S}$ & $\mathrm{H}$ & $\mathrm{R}$ & $\mathrm{L}$ & $\mathrm{K}$ \\
\hline $\mathrm{E}$ & 1 & 1 & 1 & 2 & 4 & 0 & 0 & 2 \\
$\mathrm{~T}$ & 0 & 0 & 2 & 0 & 0 & 5 & 1 & 1 \\
$\mathrm{I}$ & 0 & 1 & 0 & 3 & 2 & 0 & 1 & 0 \\
$\mathrm{~S}$ & 4 & 1 & 0 & 0 & 0 & 0 & 2 & 0 \\
$\mathrm{H}$ & 0 & 1 & 3 & 2 & 1 & 0 & 0 & 0 \\
$\mathrm{R}$ & 4 & 0 & 0 & 0 & 0 & 0 & 0 & 0 \\
$\mathrm{~L}$ & 2 & 1 & 1 & 0 & 0 & 0 & 0 & 0 \\
$\mathrm{~K}$ & 0 & 3 & 0 & 0 & 0 & 0 & 0 & 0
\end{tabular}

The first step in Jakobsen's hill climb attack is to swap the first two elements of the key. Swapping the first two elements in (4) yields a new putative key, which in this case is given by

$\begin{array}{rllllllll}\text { Plaintext: } & \text { E } & \text { T } & \text { I } & \text { S } & \text { H } & \text { R } & \text { L } & \text { K } \\ \text { Ciphertext: } & \text { R } & \text { K } & \text { I } & \text { L } & \text { H } & \text { S } & \text { E } & \text { T }\end{array}$

Applying this key to the ciphertext in (3), we find that the corresponding putative plaintext is given by

\section{EIHISTHILERTISLTTSTKERTHEIHISTHSELIEKERTSTKERTHHSLTER}

and the digram frequency matrix is given by

\begin{tabular}{c|c|c|ccccccc} 
& $\mathrm{E}$ & $\mathrm{T}$ & $\mathrm{I}$ & $\mathrm{S}$ & $\mathrm{H}$ & $\mathrm{R}$ & $\mathrm{L}$ & $\mathrm{K}$ \\
\hline $\mathrm{E}$ & 0 & 0 & 2 & 0 & 0 & 5 & 1 & 1 \\
\cline { 2 - 8 } $\mathrm{T}$ & 1 & 1 & 1 & 2 & 4 & 0 & 0 & 2 \\
\cline { 2 - 8 } $\mathrm{I}$ & 1 & 0 & 0 & 3 & 2 & 0 & 1 & 0 \\
$\mathrm{~S}$ & 1 & 4 & 0 & 0 & 0 & 0 & 2 & 0 \\
$\mathrm{H}$ & 1 & 0 & 3 & 2 & 1 & 0 & 0 & 0 \\
$\mathrm{R}$ & 0 & 4 & 0 & 0 & 0 & 0 & 0 & 0 \\
$\mathrm{~L}$ & 1 & 2 & 1 & 0 & 0 & 0 & 0 & 0 \\
$\mathrm{~K}$ & 3 & 0 & 0 & 0 & 0 & 0 & 0 & 0
\end{tabular}

Comparing the matrices in (5) and (6) we see that (6) can be obtained from (5) by simply swapping the first two rows and columns - the boxed rows and columns in (6). That is, swapping letters in the putative key, recomputing the putative plaintext, and recomputing the digram matrix is equivalent to simply swapping the corresponding rows and columns of the digram matrix. This is the crucial observation that enables the fast attack to be fast. In particular, there is no need to re-parse the ciphertext 
using the new key. Regardless of the length of the ciphertext, only a swap of rows and columns is required.

As another example, consider the key

$$
\begin{array}{rllllllll}
\text { Plaintext: } & \text { E } & \text { T } & \text { I } & \text { S } & \text { H } & \text { R } & \text { L } & \text { K } \\
\text { Ciphertext: } & \text { K } & \text { R } & \text { S } & \text { L } & \text { H } & \text { I } & \text { E } & \text { T }
\end{array}
$$

which can be obtained from (4) by swapping the third and sixth elements. In this case, the resulting digram frequencies are given by the matrix

\begin{tabular}{c|ccc|c|cc|c|cc} 
& $\mathrm{E}$ & $\mathrm{T}$ & $\mathrm{I}$ & $\mathrm{S}$ & $\mathrm{H}$ & $\mathrm{R}$ & $\mathrm{L}$ & $\mathrm{K}$ \\
\hline $\mathrm{E}$ & 1 & 1 & 0 & 2 & 4 & 1 & 0 & 2 \\
$\mathrm{~T}$ & 0 & 0 & 5 & 0 & 0 & 2 & 1 & 1 \\
\cline { 2 - 7 } $\mathrm{I}$ & 4 & 0 & 0 & 0 & 0 & 0 & 0 & 0 \\
\cline { 2 - 7 } $\mathrm{S}$ & 4 & 1 & 0 & 0 & 0 & 0 & 2 & 0 \\
$\mathrm{H}$ & 0 & 1 & 0 & 2 & 1 & 3 & 0 & 0 \\
$\mathrm{nyyyyyyyn} \mathrm{R}$ & 0 & 1 & 0 & 3 & 2 & 0 & 1 & 0 \\
\cline { 2 - 7 } $\mathrm{L}$ & 2 & 1 & 0 & 0 & 0 & 1 & 0 & 0 \\
$\mathrm{~K}$ & 0 & 3 & 0 & 0 & 0 & 0 & 0 & 0
\end{tabular}

which is obtained by simply swapping the third and sixth rows and columns of (5).

As discussed above, we compare the digram matrix $D$ to a matrix $E$ that contains expected digram values for English. That is, for each putative key, we obtain the corresponding digram matrix $D$ by swapping the appropriate rows and columns, and compute the score $d(D, E)$, using the distance function given in (2). If the swap improves the score (i.e., the distance between $D$ and $E$ decreases), then we retain the swap; otherwise we do not. In either case, we continue with the next swap, following the swapping pattern in (1).

Pseudo-code for Jakobsen's fast algorithm appears in Table 4. Note that only a single decryption of the ciphertext $C$ is performed.

How fast is the fast algorithm in Table 4? Figure 4 gives an empirical comparison between the fast algorithm and the naïve approach, where the ciphertext is re-parsed after each modification of the putative key. This particular implementation of the naïve algorithm mimics the fast algorithm, in the sense that we swap pairs of letters following the same pattern as in Jakobsen's algorithm.

The work for the naïve algorithm in Figure 4 is on the order of $N\left(\begin{array}{c}26 \\ 2\end{array}\right)$, where $N$ is the length of the ciphertext. In contrast, the work for Jakobsen's algorithm is $N+\left(\begin{array}{c}26 \\ 2\end{array}\right) \approx\left(\begin{array}{c}26 \\ 2\end{array}\right)$. That is, the work required for the fast algorithm is essentially independent of the ciphertext length, unless $N$ is large. However, if $N$ is large, we might not need to use all of the ciphertext to recover the key.

The algorithm in Figure 4 is a hill climbing attack, since we ignore any modification to the key that does not improve the score. However, the result can depend on the order in which the swaps occur. Therefore, additional swaps beyond the $\left(\begin{array}{c}26 \\ 2\end{array}\right)$ 
Table 4: Fast Simple Substitution Attack [7]

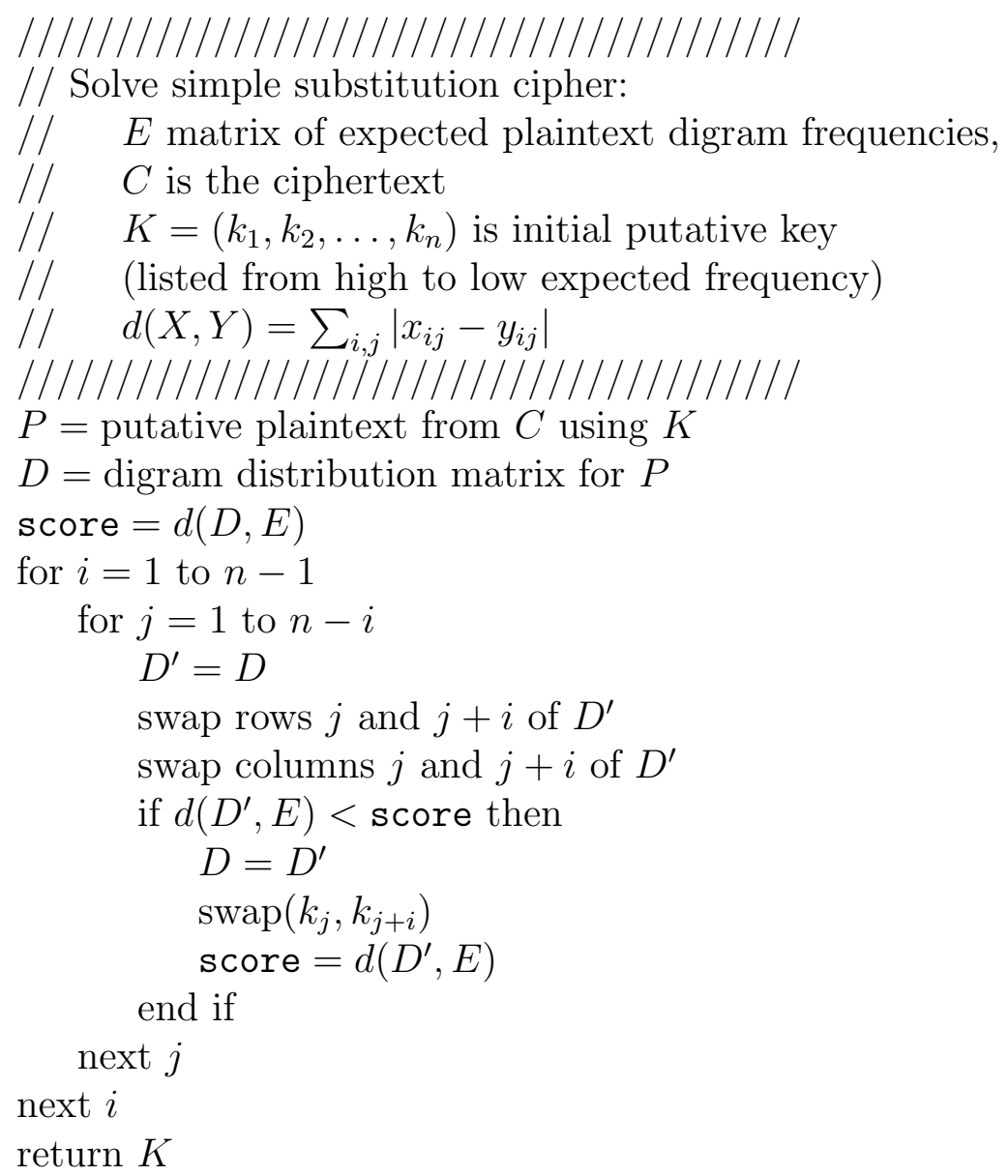

required by the algorithm could yield improved results. We return to this issue in Section 6 where we discuss attacks on the Zodiac ciphers.

In the next section, we present our fast algorithm for attacking homophonic substitution ciphers. Part of our algorithm can be considered a generalization of Jakobsen's algorithm. However, the homophonic substitution presents some unique challenges.

\section{Fast Attack on Homophonic Substitution}

As in the previous section, we assume that the plaintext in English. However, for a homophonic substitution, multiple ciphertext symbols can represent a single plaintext letter and, consequently, we need more than 26 ciphertext symbols. For a given 


\begin{tabular}{|c|c|c|}
\hline $\begin{array}{c}\text { Ciphertext } \\
\text { Size }\end{array}$ & $\begin{array}{c}\text { Statistical } \\
\text { frequency } \\
\text { algorithm }\end{array}$ & Fast \\
\hline 300 & 77 & 43 \\
\hline 500 & 71 & 34 \\
\hline 700 & 108 & 36 \\
\hline 1000 & 121 & 40 \\
\hline 2000 & 288 & 51 \\
\hline 3000 & 345 & 40 \\
\hline 4000 & 455 & 46 \\
\hline
\end{tabular}

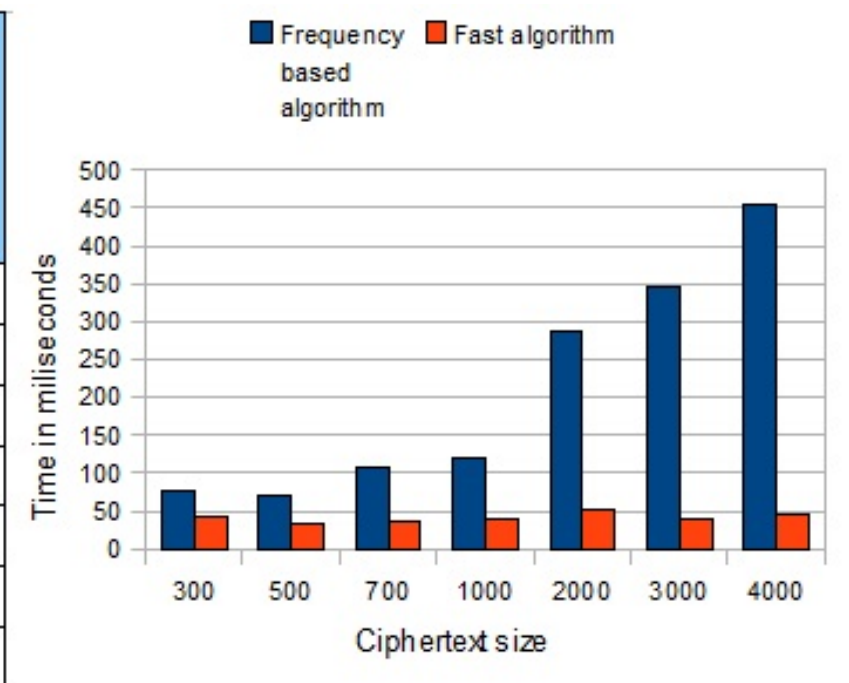

Figure 4: Naïve versus Fast Simple Substitution Attacks

homophonic substitution cipher, let $n$ be the number of ciphertext symbols. Then we have $n \geq 26$ and the special case where $n=26$ is a simple substitution. In this section, we denote the $n$ ciphertext symbols as $1,2,3, \ldots, \mathrm{n}$. Furthermore, let $n_{a}$ be the number of ciphertext symbols that correspond to plaintext $\mathrm{A}$, let $n_{b}$ be the number of symbols that correspond to B, and so on. Then

$$
n_{a}+n_{b}+n_{c}+\cdots+n_{z}=n .
$$

Our algorithm consists of following three "layers:"

- Inner hill climb layer

- Random key layer

- Outer hill climb layer

In the outer hill climb, we determine values $n_{a}, n_{b}, \ldots, n_{z}$ subject to the constraint that $n_{a}+n_{b}+\cdots+n_{z}=n$. Then in the random key layer, we generate multiple random initial keys, subject to the constraint from the outer hill climb layer, namely, that the distribution of ciphertext symbols satisfy $n_{a}, n_{b}, \ldots, n_{z}$. Finally, for the inner hill climb we employ a generalization of Jakobsen's algorithm to hill climb to a putative key, beginning with an initial key from the random key layer. That is, in the random key layer we determine a specific subset of $n_{a}$ ciphertext symbols that map to $\mathrm{A}$, a disjoint subset of $n_{b}$ ciphertext symbols that map to $\mathrm{B}$, and so on. Then in the inner hill climb layer, we improve on this solution, without altering any of the numeric values $n_{a}, n_{b}, \ldots, n_{z}$. The outer hill climb is the only layer where the numbers $n_{a}, n_{b}, \ldots, n_{z}$ change. 
These layers are nested - for each iteration of the outer hill climb, we choose multiple initial keys, and for each of these initial keys we conduct the inner hill climb. A high-level view of the connection between the layers is given in Figure 5 . Next, we discuss each of the layers in more detail.

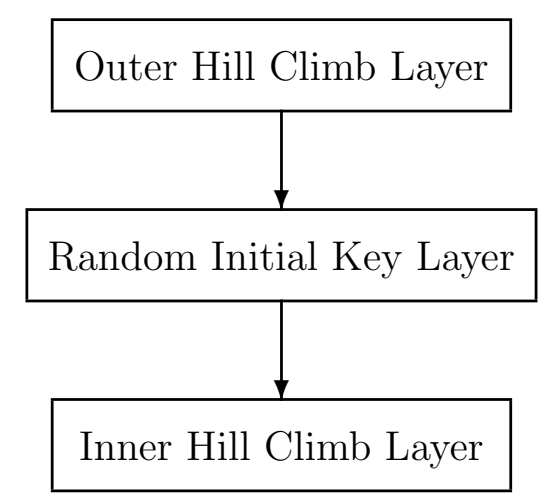

Figure 5: High Level Design

First, we discuss the inner hill climb, which can be viewed as a generalization of Jakobsen's algorithm. Here, we emphasize the similarities to (and differences from) the simple substitution case. After covering the inner hill climb in detail, we then discuss the random key layer and, finally, the outer hill climb. Both of these outer two layers are relatively simple.

\subsection{Inner Hill Climb Layer}

In this section, we assume that there are $n$ distinct ciphertext symbols and we are given the number of these symbols that map to each plaintext letter. That is, we are given $n_{a}, n_{b}, \ldots, n_{z}$ with $n_{a}+n_{b}+\cdots+n_{z}=n$. Our goal is to develop a hill climb technique, analogous to Jakobsen's algorithm, that will yield a relatively high-scoring solution to the homophonic substitution. Note that any solution at this phase is subject to the constraint that $n_{a}$ ciphertext symbols map to A, $n_{b}$ symbols map to $\mathrm{B}$, and so on. That is, the values of $n_{a}, n_{b}, n_{c}, \ldots, n_{z}$ do not change at this layer. Below, we discuss the outer hill climb, where $n_{a}, n_{b}, \ldots, n_{z}$ are specified.

As with the simple substitution attack in the previous section, we need to consider the following questions:

- How to choose the initial putative key (or keys)?

- How to modify a putative key?

- How to compute a score for a putative key? 
We consider each of these questions in turn, comparing and contrasting our approach to the simple substitution attack discussed in the previous section.

Choose the initial key: In the outer hill climb layer, we determine $n_{a}, n_{b}, \ldots, n_{z}$ with $n_{a}+n_{b}+\cdots+n_{z}=n$. Note that this does not completely specify a putative key, since we still need to determine which (disjoint) subsets of $n_{a}, n_{b}, \ldots, n_{z}$ ciphertext symbols map to A, B, . , Z, respectively. Determining such a mapping is the function of the random initial key layer, so we put off a discussion of the details of this process to Section 4.2, below.

Modify the putative key: Recall that for the simple substitution, we order the letters, then swap consecutive pairs, followed by pairs at distance two, and so on, as summarized in (1). In this way, all $\left(\begin{array}{c}26 \\ 2\end{array}\right)$ pairs of letters are swapped exactly once.

For the homophonic substitution, we follow an analogous process. However, letters can appear multiple times in the key and we must avoid swapping a letter with itself swapping a letter with itself would not change the putative plaintext. Consider the example in Table 5, where the ciphertext symbols are $0,1, \ldots, 9$ and the initial key maps $\mathrm{E}$ and $\mathrm{T}$ to two ciphertext symbols each, with all remaining letters mapped to a single ciphertext symbol. In Table 5, the boxed elements in each row are swapped. For simplicity, we assume that the score does not increase for any of the swaps considered, that is, the swaps do not affect the putative key.

Table 5: Modification of Putative Key

\begin{tabular}{ccccccccccc}
\hline Ciphertext & $\mathrm{O}$ & 1 & 2 & 3 & 4 & 5 & 6 & 7 & 8 & 9 \\
\hline Initial key & $\mathrm{S}$ & $\mathrm{H}$ & $\mathrm{I}$ & $\mathrm{E}$ & $\mathrm{E}$ & $\mathrm{T}$ & $\mathrm{T}$ & $\mathrm{K}$ & $\mathrm{R}$ & $\mathrm{L}$ \\
\hline 1st swap & $\mathrm{S}$ & $\mathrm{H}$ & $\mathrm{I}$ & $\mathrm{E}$ & $\mathrm{E}$ & $\mathrm{T}$ & $\mathrm{T}$ & $\mathrm{K}$ & $\mathrm{R}$ & $\mathrm{L}$ \\
2nd swap & $\mathrm{S}$ & $\mathrm{H}$ & $\mathrm{I}$ & $\mathrm{E}$ & $\mathrm{E}$ & $\mathrm{T}$ & $\mathrm{T}$ & $\mathrm{K}$ & $\mathrm{R}$ & $\mathrm{L}$ \\
3rd swap & $\mathrm{S}$ & $\mathrm{H}$ & $\mathrm{I}$ & $\mathrm{E}$ & $\mathrm{E}$ & $\mathrm{T}$ & $\mathrm{T}$ & $\mathrm{K}$ & $\mathrm{R}$ & $\mathrm{L}$ \\
4th swap & $\mathrm{S}$ & $\mathrm{H}$ & $\mathrm{I}$ & $\mathrm{E}$ & $\mathrm{E}$ & $\mathrm{T}$ & $\mathrm{T}$ & $\mathrm{K}$ & $\mathrm{R}$ & $\mathrm{L}$ \\
5th swap & $\mathrm{S}$ & $\mathrm{H}$ & $\mathrm{I}$ & $\mathrm{E}$ & $\mathrm{E}$ & $\mathrm{T}$ & $\mathrm{T}$ & $\mathrm{K}$ & $\mathrm{R}$ & $\mathrm{L}$ \\
6th swap & $\mathrm{S}$ & $\mathrm{H}$ & $\mathrm{I}$ & $\mathrm{E}$ & $\mathrm{E}$ & $\mathrm{T}$ & $\mathrm{T}$ & $\mathrm{K}$ & $\mathrm{R}$ & $\mathrm{L}$ \\
7th swap & $\mathrm{S}$ & $\mathrm{H}$ & $\mathrm{I}$ & $\mathrm{E}$ & $\mathrm{E}$ & $\mathrm{T}$ & $\mathrm{T}$ & $\mathrm{K}$ & $\mathrm{R}$ & $\mathrm{L}$ \\
8th swap & $\mathrm{S}$ & $\mathrm{H}$ & $\mathrm{I}$ & $\mathrm{E}$ & $\mathrm{E}$ & $\mathrm{T}$ & $\mathrm{T}$ & $\mathrm{K}$ & $\mathrm{R}$ & $\mathrm{L}$ \\
$\vdots$ & $\vdots$ & $\vdots$ & $\vdots$ & $\vdots$ & $\vdots$ & $\vdots$ & $\vdots$ & $\vdots$ & $\vdots$ & $\vdots$ \\
\hline \hline
\end{tabular}

Consider the "3rd swap" and "4th swap" rows in Table 5 . Note that we swap the I in column 2 with the $\mathrm{E}$ in column 3 and with the $\mathrm{E}$ in column 4 . That is, we must swap I with both E columns, since they correspond to different (in fact, disjoint) sets 
of symbols. However, we do not swap the $\mathrm{E}$ in column 4 with the $\mathrm{E}$ in column 5 . The score is based on English letters, so replacing E with E would not change the score.

The number of swaps required depends on $n$ and the values of $n_{a}, n_{b}, \ldots, n_{z}$. Let $m_{i}$, for $i=1,2, \ldots, 26$ be the values of $n_{a}, n_{b}, \ldots, n_{z}$, respectively. Then each of $m_{1}$ identical letters is swapped with all $n-m_{1}$ other letters, each of the $m_{2}$ identical letters is swapped with all remaining $n-m_{1}-m_{2}$ letters and so on. Thus, the total number of swaps is given by

$$
\sum_{i=1}^{26} m_{i}\left(n-\sum_{j=1}^{i} m_{j}\right)=n^{2}-\sum_{i=1}^{26} m_{i} \sum_{j=1}^{i} m_{j}=\sum_{i=1}^{25} \sum_{j=i+1}^{26} m_{i} m_{j} .
$$

In practice, we consider all $\left(\begin{array}{l}n \\ 2\end{array}\right)$ pairs of ciphertext symbols, following a scheme analogous to that given in (1), and we simply skip any pairs that currently map to the same plaintext letter. The number of these $\left(\begin{array}{l}n \\ 2\end{array}\right)$ pairs that will require a swap and score computation is given by (7). Consequently, a crude upper bound on (7) is given by $\left(\begin{array}{l}n \\ 2\end{array}\right)$.

Compute the score: Recall that for the fast attack on a simple substitution, we have two matrices $D$ and $E$, both of which are $26 \times 26$. The $E$ matrix contains expected digram frequencies for English, while the $D$ matrix contains digram frequencies corresponding to the putative plaintext. The algorithm is fast, since a swap of elements in the putative key only requires a swap of the corresponding rows and columns of the $D$ matrix. For the simple substitution attack, we compute the score by measuring the distance between matrices $D$ and $E$ using the formula in (2).

The same principle can be applied to the homophonic substitution, but the situation is complicated by the fact that there are more ciphertext symbols than plaintext symbols. That is, for a homophonic substitution, in general, we have $n>26$.

For the homophonic substitution, we still use a fixed $E$ matrix containing the expected English digram frequencies. But, instead of a single $D$ matrix, we employ two matrices to represent the digram frequencies. Let $D_{C}$ be an $n \times n$ matrix containing ciphertext digram frequencies. This matrix is constructed only once, and never changes.

The second digram distribution matrix, which we denote as $D_{P}$, is of size $26 \times 26$ and contains the letter digram frequencies corresponding to the current putative plaintext. This matrix corresponds to the $D$ matrix in the simple substitution attack.

As in simple substitution attack, the $D_{P}$ matrix is modified with each swap of elements in the putative key. However, it is not sufficient to simply swap rows and columns of $D_{P}$. Instead, we use the ciphertext digram frequencies in $D_{C}$ to help determine the correct elements for the affected rows and columns of $D_{P}$. Once we have determined $D_{P}$, the score computation is the same as in the simple substitution case, that is, we compute score $=d\left(D_{P}, E\right)$.

To illustrate the process used to update the $D_{P}$ matrix, we consider a simplified example. As in the examples in Section 3, suppose that our plaintext alphabet is 
restricted to the eight letters

E, T, I, S, H, R, L, and K.

Consider a homophonic substitution, where the plaintext is restricted to these eight letters, and where there are 10 ciphertext symbols, denoted $0,1, \ldots, 9$.

Suppose that we are given the ciphertext

09498249507298522861072309398248059010768610764485207.

Then we construct the $10 \times 10$ ciphertext digram matrix $D_{C}$,

\begin{tabular}{l|llllllllll} 
& 0 & 1 & 2 & 3 & 4 & 5 & 6 & 7 & 8 & 9 \\
\hline 0 & 0 & 1 & 0 & 0 & 0 & 1 & 0 & 5 & 0 & 2 \\
1 & 3 & 0 & 0 & 0 & 0 & 0 & 0 & 0 & 0 & 0 \\
2 & 1 & 0 & 1 & 1 & 2 & 0 & 0 & 0 & 1 & 1 \\
3 & 1 & 0 & 0 & 0 & 0 & 0 & 0 & 0 & 0 & 1 \\
4 & 0 & 0 & 0 & 0 & 1 & 0 & 0 & 0 & 2 & 2 \\
5 & 1 & 0 & 2 & 0 & 0 & 0 & 0 & 0 & 0 & 1 \\
6 & 0 & 2 & 0 & 0 & 1 & 0 & 0 & 0 & 1 & 0 \\
7 & 0 & 0 & 2 & 0 & 0 & 0 & 2 & 0 & 0 & 0 \\
8 & 1 & 0 & 2 & 0 & 0 & 2 & 2 & 0 & 0 & 0 \\
9 & 1 & 0 & 0 & 1 & 1 & 1 & 0 & 0 & 3 & 0
\end{tabular}

Next, suppose that from the outer hill climb (discussed below), we are given the constraint that two ciphertext symbols map to $\mathrm{E}$ and two symbols map to $\mathrm{T}$, with one symbol mapping to each of the remaining six characters. With this constraint, suppose that we construct the following initial putative key:

$$
\begin{array}{c|cccccccccc}
\text { ciphertext } & 0 & 1 & 2 & 3 & 4 & 5 & 6 & 7 & 8 & 9 \\
\hline \text { plaintext } & \text { I } & \text { L } & \text { E } & \text { K } & \text { T } & \text { E } & \text { R } & \text { T } & \text { H } & \text { S }
\end{array}
$$

Note that the encryption version of this key can be written as

\begin{tabular}{r|cccccccc} 
plaintext & $\mathrm{E}$ & $\mathrm{T}$ & $\mathrm{I}$ & $\mathrm{S}$ & $\mathrm{H}$ & $\mathrm{R}$ & $\mathrm{L}$ & $\mathrm{K}$ \\
\hline ciphertext & 2 & 4 & 0 & 9 & 8 & 6 & 1 & 3 \\
& 5 & 7 & & & & & &
\end{tabular}

That is, there are two ciphertext symbols that can be substituted for E (either 2 or 5 ) and two choices for $\mathrm{T}$ (either 4 or 7 ), while the remaining plaintext letters each have a single corresponding ciphertext value. For the attack, we use the decryption key which, for this example, we denote as ILEKTERTHS.

Applying the putative key ILEKTERTHS to the ciphertext in (8), we obtain the following putative plaintext:

ISTSHETSEITESHEEEHRLITEKISKSHETHIESILITRHRLITRTTHEEIT. 
We can use this putative plaintext to generate the digram frequency matrix $D_{P}$ which, in this case, is given by

\begin{tabular}{c|cccccccc} 
& $\mathrm{E}$ & $\mathrm{T}$ & $\mathrm{I}$ & $\mathrm{S}$ & $\mathrm{H}$ & $\mathrm{R}$ & $\mathrm{L}$ & $\mathrm{K}$ \\
\hline $\mathrm{E}$ & 3 & 2 & 2 & 2 & 1 & 0 & 0 & 1 \\
$\mathrm{~T}$ & 2 & 1 & 0 & 2 & 2 & 2 & 0 & 0 \\
$\mathrm{I}$ & 1 & 5 & 0 & 2 & 0 & 0 & 1 & 0 \\
$\mathrm{~S}$ & 1 & 1 & 1 & 0 & 3 & 0 & 0 & 1 \\
$\mathrm{H}$ & 4 & 0 & 1 & 0 & 0 & 2 & 0 & 0 \\
$\mathrm{R}$ & 0 & 1 & 0 & 0 & 1 & 0 & 2 & 0 \\
$\mathrm{~L}$ & 0 & 0 & 3 & 0 & 0 & 0 & 0 & 0 \\
$\mathrm{~K}$ & 0 & 0 & 1 & 1 & 0 & 0 & 0 & 0
\end{tabular}

In (11) we have listed the letters in order from highest expected frequency to lowest.

As with the simple substitution attack, at this point, we modify the putative key by swapping elements, and after each swap, we update the putative plaintext digram matrix, $D_{P}$ and compute the score by comparing the updated matrix with the expected English digram matrix $E$. If the score improves, we update the putative key; if not, we leave the key unchanged.

For the simple substitution, updating the plaintext digram matrix $D$ was accomplished by swapping the relevant rows and columns. For the homophonic substitution, updating $D_{P}$ is slightly more involved, and we will need to make use of the matrix $D_{C}$.

Next, we illustrate the process used to update $D_{P}$ by considering two examples. For the first case, suppose that in the decryption key ILEKTERTHS, we swap L and K, that is

$$
\text { ILEKTERTHS } \rightarrow \text { IKELTERTHS. }
$$

Since neither K nor L corresponds to multiple ciphertext symbols, this case is identical to swapping letters in the simple substitution. Therefore, we simply swap the $\mathrm{K}$ and $\mathrm{L}$ rows and columns of $D_{P}$, that is, we follow the same procedure as in the simple substitution attack.

The difficulty arises when one (or both) of the swapped letters correspond to more than one ciphertext symbol. For example, suppose we swap S with the first E in the putative key, that is,

$$
\text { ILEKTERTHS } \rightarrow \text { ILSKTERTHE. }
$$

Then we cannot simply swap the corresponding rows (and columns) or $D_{P}$, since the E row (and column) includes counts for both of the $E$ letters that appear in the key, and we have only swapped one of the them.

Decrypting the ciphertext using the modified key in (12), we obtain the putative plaintext

IETEHSTEEITSEHESSHRLITSKIEKEHSTHIEEILITRHRLITRTTHESIT. 
From this putative plaintext, we tabulate the digram frequencies to obtain the following array (the affected rows and columns are boxed):

\begin{tabular}{|c|c|c|c|c|c|c|c|c|}
\hline & $\mathrm{E}$ & $\mathrm{T}$ & I & $\mathrm{S}$ & $\mathrm{H}$ & $\mathrm{R}$ & $\mathrm{L}$ & K \\
\hline$E$ & 2 & 1 & 2 & 2 & 3 & 0 & 0 & 1 \\
\hline $\mathrm{T}$ & 2 & 1 & 0 & 2 & 2 & 2 & 0 & $\overline{0}$ \\
\hline$I$ & 3 & 5 & 0 & 0 & 0 & 0 & 1 & 0 \\
\hline$S$ & 1 & 2 & 1 & 1 & 1 & 0 & 0 & 1 \\
\hline $\mathrm{H}$ & 2 & 0 & 1 & 2 & 0 & 2 & 0 & 0 \\
\hline $\mathrm{R}$ & 0 & 1 & 0 & 0 & 1 & 0 & 2 & 0 \\
\hline $\mathrm{L}$ & 0 & 0 & 3 & 0 & 0 & 0 & 0 & 0 \\
\hline $\mathrm{K}$ & 1 & 0 & 1 & 0 & 0 & 0 & 0 & 0 \\
\hline
\end{tabular}

Note that elements outside of the $\mathbf{E}$ and $\mathbf{S}$ rows and columns are identical in (11) and (13). This is as expected, since those letters were not involved in the swapping. However, if we simply swap the $\mathrm{E}$ and $\mathrm{S}$ rows (and columns) in (11) we do not obtain the frequencies in (13). Swapping the appropriate rows and columns of (11), would give us a 0 in the $\mathrm{HE}$ position (i.e., in row $\mathrm{H}$ and column $\mathrm{E}$ ). However, in (13) we find a 2 in the HE position. Also, swapping, would yield 0 in the upper left EE position, but in (13) we observe that EE has a count of 2 .

To resolve the elements in the swapped rows and columns, we employ the $D_{C}$ matrix in (9). From the putative key in (10), we note that the swapped elements E and $\mathrm{S}$ correspond to the rows (and columns) labeled 2 and 9, respectively. Consider the count for HE in matrix $D_{P}$. Before the swap, the $\mathrm{H}$ in $\mathrm{HE}$ corresponds to row 8 (since plaintext $\mathrm{H}$ is encrypted as ciphertext 8), while the $\mathrm{E}$ correspond to either column 2 or 5 . In $D_{C}$ we have a 2 in position 82 (i.e., row 8 , column 2) and a 2 in position 85 giving a total of 4 . This agrees with the pre-swap matrix $D_{P}$ in (11), which has a 4 in position HE.

Swapping columns 2 and 9 of $D_{C}$, would put a 0 into position 82, leaving the 2 in position 85 unchanged. Therefore, post-swap, the $D_{P}$ matrix will have a 2 in element HE, which agrees with (13).

In practice, we do not actually perform any swaps in $D_{C}$. Instead, we can simply examine the elements that would have been swapped. Since the key elements are swapped (in cases where the score improves), the correspondence between elements of the putative key and the rows and columns of $D_{C}$ will be maintained.

The EE case mentioned above is more complex, since we need to consider four cases, and both the row and column swaps are relevant. We leave the details of this case to the reader.

The bottom line is that we can use the $D_{C}$ matrix to fill in the elements in the swapped rows and columns. In the process, we do not perform any swaps on the $D_{C}$ matrix itself. The elements in the putative key are swapped as appropriate, so the $D_{C}$ matrix remains valid for subsequent iterations. Also, it is worth noting that there are 
symmetries that can be exploited, which reduce the number of elements that must be computed using the $D_{C}$ matrix by at least half. ${ }^{1}$ Finally, note that given any putative key $K$, we can use the $D_{C}$ matrix to determine the corresponding $D_{P}$ matrix. That is, the use of $D_{C}$ to compute $D_{P}$ is not limited to swapping elements of the key-for arbitrary changes to the key, we can use $D_{C}$ to determine all values of $D_{P}$. We make use of this fact in the random initial key layer, which we discuss next.

\subsection{Random Initial Key Layer}

Recall that for the simple substitution attack, we choose an initial key that gives the best match between ciphertext and English letter frequencies. Consequently, for the case of a simple substitution, it is trivial to determine an initial putative key.

For our homophonic substitution attack, the analogous step would be to chose an initial key that best matches ciphertext letter frequencies of English letter frequencies, subject to the outer hill climb constraints, $n_{a}, n_{b}, \ldots, n_{z}$. However, finding an optimal solution to this problem is decidedly nontrivial, since there are, in general, a large number of cases to consider. For simplicity, suppose that all plaintext letters are mapped to more than one ciphertext symbol. In this case, the number of possible initial key combinations is given by the multinomial coefficient

$$
\left(\begin{array}{c}
n \\
n_{a}, n_{b}, \ldots, n_{z}
\end{array}\right)=\frac{n !}{n_{a} ! n_{b} ! \cdots n_{z} !} .
$$

In the more general case, where some of the plaintext letters are mapped to a single ciphertext symbol, the formula is slightly more complicated. Suppose that $\ell$ of the plaintext letters are each mapped to a single ciphertext symbol. For these letters we can do no better than simply choosing a ciphertext symbol that most closely matches the expected English frequency. Then each of the remaining $n-\ell$ plaintext letters corresponds to more than one ciphertext symbol. Let $m_{1}, m_{2}, \ldots, m_{n-\ell}$ be the elements of the set $\left\{n_{a}, n_{b}, n_{c}, \ldots, n_{z}\right\}$ that are greater than one. The number of combinations we must consider is

$$
\left(\begin{array}{c}
n-\ell \\
m_{1}, m_{2}, \ldots, m_{n-\ell}
\end{array}\right)=\frac{(n-\ell) !}{m_{1} ! m_{2} ! \cdots m_{n-\ell} !}
$$

\footnotetext{
${ }^{1}$ When swapping putative key elements, ciphertext is conserved, that is, no characters are inserted or deleted. Consequently, any differences between the correct $D_{P}$ and the results obtained by simply swapping elements (as in the simple substitution attack) must sum to zero between "swapped" pairs. For example, above we noted that if we swap the first $\mathrm{E}$ row (and column) with the $\mathbf{S}$ row (and column) in (11), then the HE entry would be 0 . However, we see in (13) that the correct value is 2 . That is, the correct value is 2 more than the swapped value. The corresponding element to HE is HS, that is, the values in HE and HS are swapped in the simple substitution attack. If these values were swapped, then HS would be 4 in $D_{P}$, but it is actually 2, which is 2 less than the number obtained by swapping. In general, these differences in "swap pairs" must sum to 0 . So, once we compute HE, we can determine HS by reference to its swap value and HE, without any need to reference $D_{C}$. Similarly, EE is 2 greater than the value obtained by swapping, and its corresponding element of SS is 1, which is indeed 2 less than we would obtain by swapping.
} 
Note that in the case of a simple substitution, $\ell=n$ and the formula in (14) yields 1 . That is, (14) holds for the simple substitution.

Since we cannot hope to choose an optimal initial key, we use a simple greedy approach. Specifically, we find subsets that approximate the letter frequencies, beginning with the highest-frequency letters and proceeding to the lower frequency letters. That is, we first find a subset of size $n_{e}$ that is a reasonable approximation to the expected frequency of $\mathrm{E}$, followed by a subset of size $n_{t}$ that approximates the expected frequency of $\mathrm{T}$, followed by a subset of size $n_{a}$ that approximates the frequency of A in English text, and so on.

For example, suppose that $n_{e}=n_{t}=2$ and $n_{a}=1$, and we have the ciphertext frequencies in Table 6 . We see that ciphertext symbols 4 and 5 can be combined to give a good approximation (12.9\%) to the expected frequency of plaintext letter $\mathrm{E}$ $(12.7 \%)$. Similarly, symbols 6 and 7 together approximate the expected frequency of $\mathrm{T}$ while symbol 1 approximates the expected frequency of A. Note that this approach will generally find good approximations for the higher frequency letters, but may not be as accurate for the lower frequency letters. This is a reasonable compromise between efficiency and accuracy, since the high frequency letters will have the largest impact on the solution.

Table 6: Initial Key

\begin{tabular}{cccccccccc}
\hline \hline Ciphertext & 0 & 1 & 2 & 3 & 4 & 5 & 6 & $\cdots$ & $\mathrm{n}$ \\
Frequency & $8.3 \%$ & $7.5 \%$ & $6.7 \%$ & $6.6 \%$ & $6.3 \%$ & $5.5 \%$ & $4.4 \%$ & $\cdots$ & $0.1 \%$ \\
\hline Initial key & $\mathrm{A}$ & $\mathrm{0}$ & $\mathrm{E}$ & $\mathrm{E}$ & $\mathrm{I}$ & $\mathrm{T}$ & $\mathrm{T}$ & $\cdots$ & $\mathrm{Q}$ \\
\hline \hline
\end{tabular}

As discussed in Section 2.4, a hill climb attack can only find a local maximum. One way to improve the results of any hill climb is to conduct the attack multiple times using different initial starting points. Therefore, in the random initial key layer we generate a series of $R$ distinct initial keys, all of which are consistent with the outer hill climb layer. That is, given $n_{a}, n_{b}, \ldots, n_{z}$ with $n_{a}+n_{b}+\cdots+n_{z}=n$, for each of the $R$ iterations of the random initial key layer, we select a subset of $n_{e}$ ciphertext symbols that map to $\mathrm{E}$, and $n_{t}$ ciphertext symbols that map to $\mathrm{T}$, and so on. For each case we use a slightly modified greedy algorithm. In practice, it is easy to find a large number of distinct initial keys.

For each of the $R$ initial putative keys generated at this layer, we first determine the corresponding $D_{P}$ (using $D_{C}$, without decrypting the ciphertext), then complete the inner hill climb. The final result of the random initial key layer is the best-scoring putative key obtained in any of these $R$ iterations over the inner hill climb.

We conducted experiments to empirically determine a useful value for $R$. These experiments are discussed in Section 5. 


\subsection{Outer Hill Climb}

In Section 4.2 we discussed the method used to generate initial keys that satisfy the constraint $n_{a}, n_{b}, \ldots, n_{z}$ where $n_{a}+n_{b}+\cdots+n_{z}=n$. The outer hill climb layer provides these constraints, that is, the outer hill climb specifies the number of ciphertext symbols that are mapped to each letter.

As the name indicates, this layer uses a hill climb approach. That is, we initially specify $n_{a}, n_{b}, \ldots, n_{z}$ with $n_{a}+n_{b}+\cdots+n_{z}=n$. Then we iterate, where at each iteration we modify these subset sizes by "swapping" adjacent pairs (ranked from high frequency to low). The swapping is somewhat different than in the inner hill climb, so we explain it in more detail below. But, the point is that for each swap we compute a score (i.e., the best score obtained via the random key and inner hill climb layers) and use this score to guide adjustments in the distribution of letters to symbols. That is, the goal of the outer hill climb is to climb to the correct distribution of ciphertext symbols to letters.

First, we need to specify an initial distribution to begin the outer hill climb. We begin by, in a sense, assuming the worst case. Specifically, we assume that the mapping of letters to ciphertext symbols was chosen to flatten the ciphertext statistics as much as possible. That is, we assume that about $12 \%$ of the symbols correspond to E, while the letter $\mathrm{T}$ corresponds to about $9 \%$ of the symbols, and so on. In addition, we assume that at least one symbol corresponds to each letter of the

alphabet. Examples of such initial distributions for various ciphertext symbol sizes appear in Table 7.

Table 7: Initial Frequency Distribution

\begin{tabular}{|c|c|c|c|c|c|c|c|c|c|c|c|c|c|c|c|c|c|c|c|c|c|c|}
\hline & \multicolumn{22}{|c|}{${ }_{h} n_{d} n_{l} n_{c} n_{u} n_{m} n_{f} n_{w} n_{g}$} \\
\hline & & & & & & & & & & 11 & & & & 1 & & & & & 1 & & & \\
\hline & & 1 & & 1 & & & & & & 11 & & & & 1 & & & & & & & & \\
\hline & 4 & 2 & 2 & 2 & 2 & 1 & & & & 11 & & & & 1 & & & & & 1 & 1 & & \\
\hline & & 3 & 3 & 3 & 3 & 2 & 2 & 1 & & $\begin{array}{ll}1 & 1\end{array}$ & & & 1 & 1 & & & & & & & & \\
\hline & & & 4 & 4 & & $\begin{array}{l}33 \\
3\end{array}$ & 3 & 2 & & 1 & & & 1 & 1 & & & & & & 1 & & \\
\hline & & & 5 & 54 & & 44 & 4 & 2 & 2 & $\begin{array}{ll}2 & 1\end{array}$ &. & & 1 & 1 & & & & & & & & \\
\hline & & & 6 & 5 & & $\begin{array}{ll}54 \\
\end{array}$ & $=4$ & 3 & & 22 & 2 & & 1 & 1 & & & & 1 & 1 & & & \\
\hline & 8 & 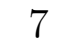 & 7 & 6 & 6 & $5 \quad 5$ & 5 & 3 & & $\begin{array}{ll}22 \\
2\end{array}$ & 2 & & 1 & 1 & & & & 1 & 1 & 1 & & \\
\hline & & & & & & & & & & & & & & & & & & & & & & \\
\hline
\end{tabular}

Next, we discuss the outer hill climb "swapping" process in more detail. Suppose that $n=35$. Then for the initial distribution, as given in Table 7 , we have $n_{e}=4$ and $n_{t}=2$ and so on. Using this distribution, we execute the random initial key layer which, in turn, calls the inner hill climb. The best score of this entire process 
is saved as the current score. Then we "swap," starting with $n_{e}$ and $n_{t}$. However, instead of simply swapping the values, we increment $n_{e}$ and decrement $n_{t}$. So, for this example, the first swap consists of setting $n_{e}=5$ and $n_{t}=1$, with the remaining values unchanged. Note that the distribution still satisfies $n_{a}+n_{b}+\cdots+n_{z}=n$, as required. Using this new distribution, we call the random initial key layer, which calls the inner hill climb. If the best score from this process is less than score, then we update score and maintain this new distribution; if not, score is unchanged and we revert to the previous distribution.

If the score does not improve, then we switch the order of the pair and "swap" again. For the example in the previous paragraph, we would increment the original $n_{t}$ and decrement the original $n_{e}$. That is, we let $n_{t}=n_{e}=3$ and execute the inner layers. Then for the next swap, we consider the pair $n_{t}$ and $n_{a}$, and so on.

We first perform this outer swap on all consecutive pairs, then all pairs at distance 2 , then all pairs at distance 3 , and so on. The process is analogous to that used in the inner hill climb and illustrated in (1). However, in this case, the order matters, since one element is incremented and the other decremented, so we have twice as many cases to consider. Again, we do not swap the actual values, but instead, we increment and decrement the counts, subject to the constraint that every count must be at least 1 . Since every count must be at least 1 , whenever the count to be decremented is 1 , we skip that step.

\subsection{All Together Now}

A high level overview of the algorithm appears in Figure 6. This diagram illustrates the relationship between the three layers.

Pseudo-code for the attack appears in Tables 8, 9 and 10. Specifically, pseudocode for the outer hill climb layer is given in Table 8, while Table 9 has pseudo-code for the random initial key layer, and Table 10 gives the pseudo-code for the inner hill climb layer.

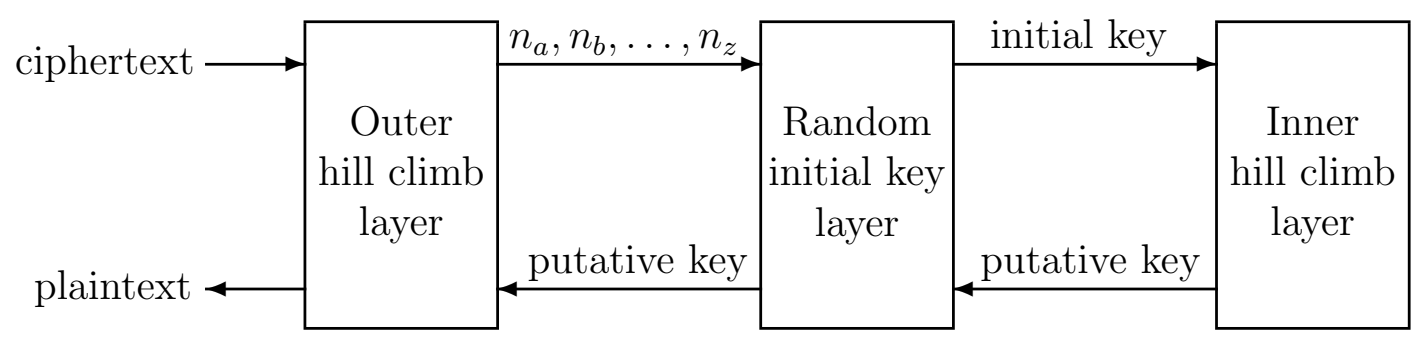

Figure 6: Block Diagram 
Table 8: Outer Hill Climb Layer

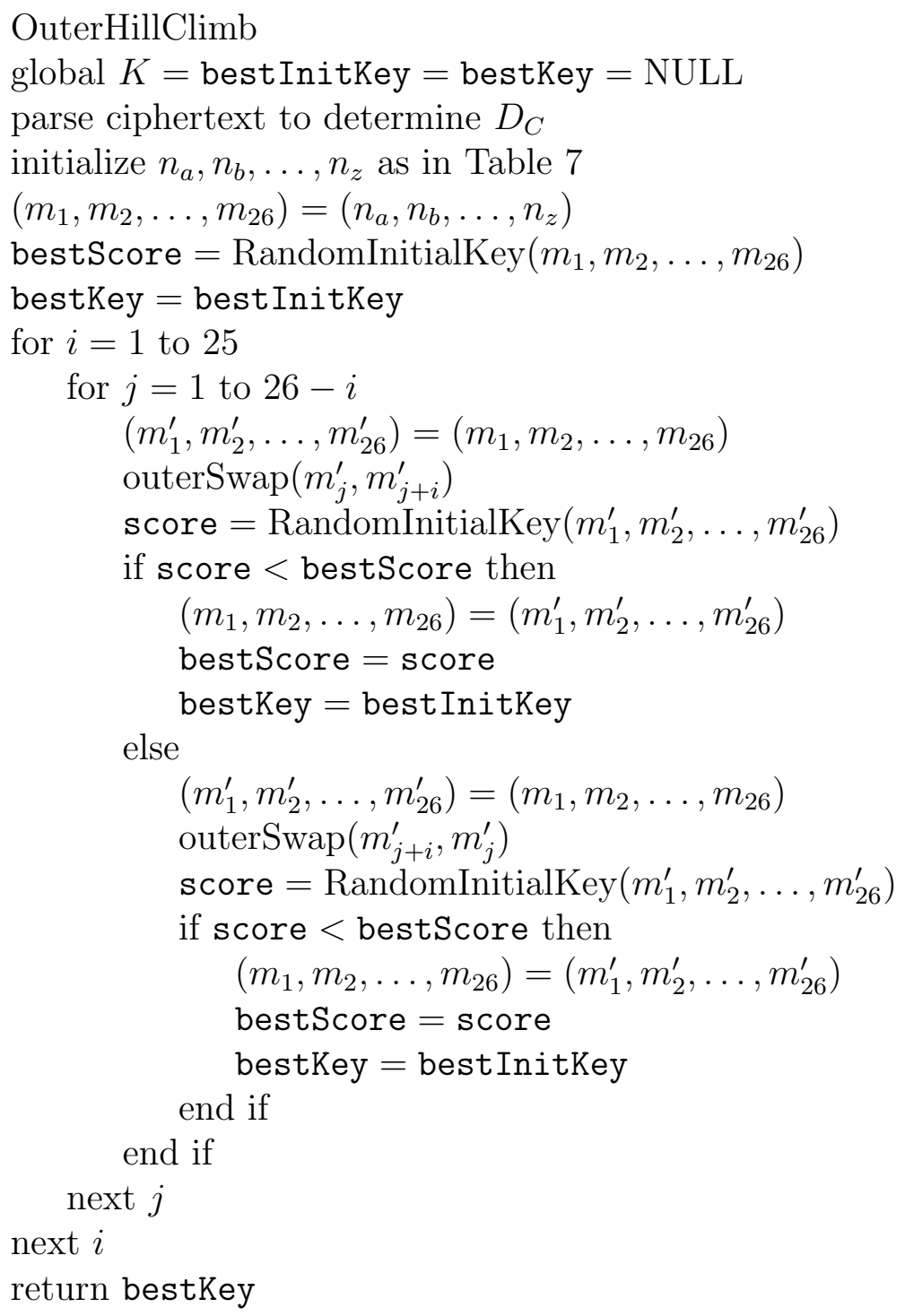

For the outer hill climb layer, the "outerSwap" function is defined as

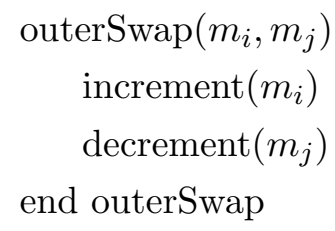


To simplify the pseudo-code for the outer hill climb that appears in Table 8, we assume that the second argument to outerSwap is greater than one. If not, we skip that particular step, since we require that at least one ciphertext symbol maps to each plaintext letter, that is, $m_{i} \geq 1$ for all $i$.

Table 9: Random Initial Key Layer

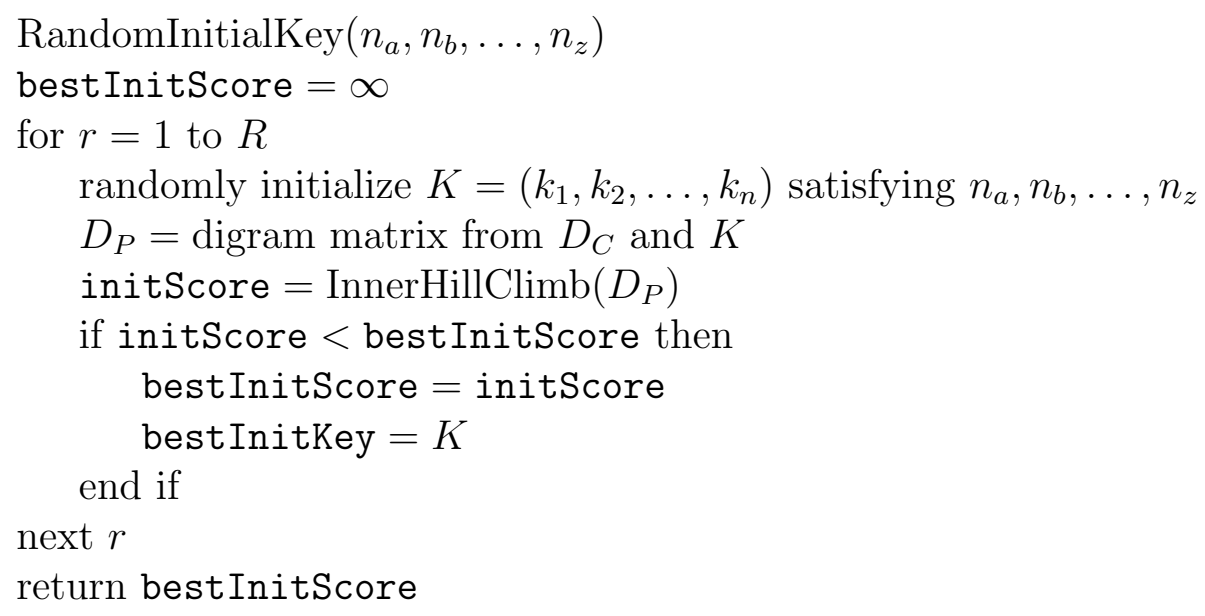

Table 10: Inner Hill Climb Layer

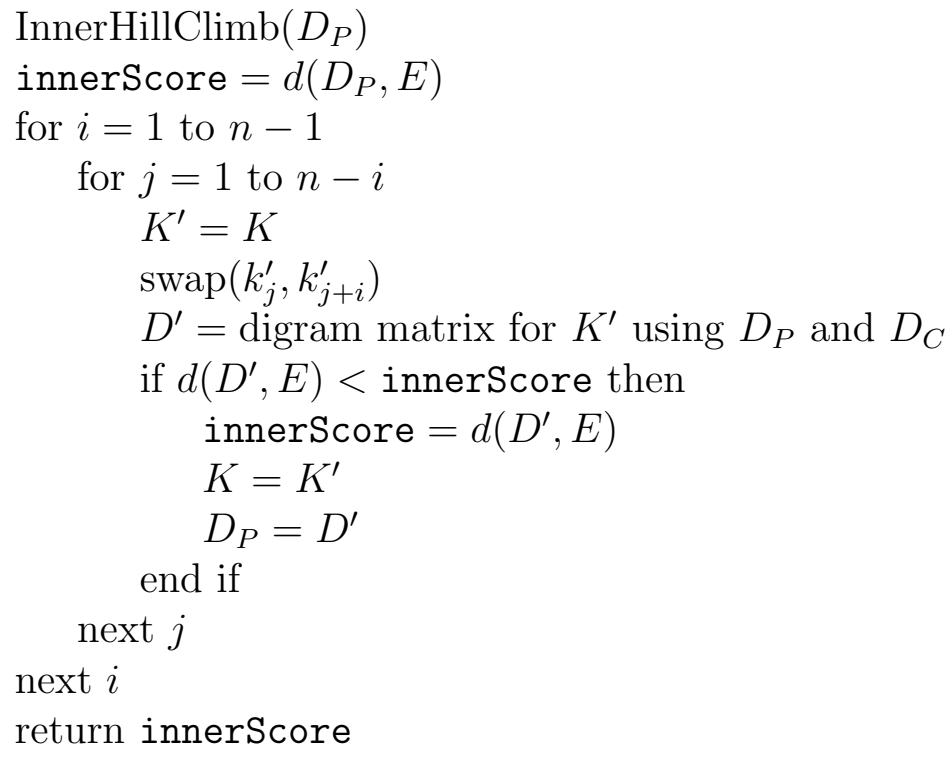




\subsection{Work Factor}

As mentioned above, an exhaustive search attack on a homophonic substitution cipher has a work factor of about $2^{4.7 n}$, where $n$ is the number of ciphertext symbols. The work factor for our hill climb attack depends on the following:

- The number of key distribution mappings generated in the outer hill climb layer: For each pair from the set $n_{a}, n_{b}, \ldots, n_{z}$ for which both counts are greater than 1 , we "swap" the counts by incrementing and decrementing, and the order matters. At most, this gives us $2\left(\begin{array}{c}26 \\ 2\end{array}\right)$ iterations of the outer hill climb layer.

- The number of initial starting points generated in the random initial key layer: Experimentally, we determined that 40 random starts works well in practice. This experiment is described in the next section.

- The number of score computations performed in the inner hill climb layer: The precise value is given in (7), but, for simplicity, we use the upper bound $\left(\begin{array}{l}n \\ 2\end{array}\right)$.

Combining these results, we find that the work factor is bounded by

$$
80 \cdot\left(\begin{array}{c}
26 \\
2
\end{array}\right) \cdot\left(\begin{array}{c}
n \\
2
\end{array}\right)<2^{15} n^{2}
$$

where this work factor is in terms of the number of score calculations of the form

$$
\text { score }=d\left(D_{P}, E\right) .
$$

The work factor in (15) ignores the one-time work of constructing the ciphertext digram matrix $D_{C}$. Constructing $D_{C}$ is the only work that depends on the ciphertext length, since no decryption of the plaintext is required. This is analogous to the fast simple substitution attack, where one initial decryption is needed, after which the algorithm has no dependence on the ciphertext length.

Neglecting the (minimal) dependence on the ciphertext length, for the simple substitution attack, the work factor is $\left(\begin{array}{c}26 \\ 2\end{array}\right)<2^{9}$, in terms of the number of score calculations. Consequently, the work for our homophonic substitution attack is on the order of $64 n^{2}$ times greater than required for the fast simple substitution attack. In addition, each score computation for the homophonic substitution is slightly more complex, since we use the $D_{C}$ matrix, as opposed to simply swapping rows and columns of the digram matrix $D_{P}$.

Timing results for our implementation of the homophonic substitution attack appear in Figure 7. These empirical timings appear to be generally consistent with the analysis in this section.

\section{$5 \quad$ Experimental Results}

We have implemented the fast homophonic substitution attack discussed in the previous section. The code was written in $\mathrm{C}++$ in a UNIX environment. 


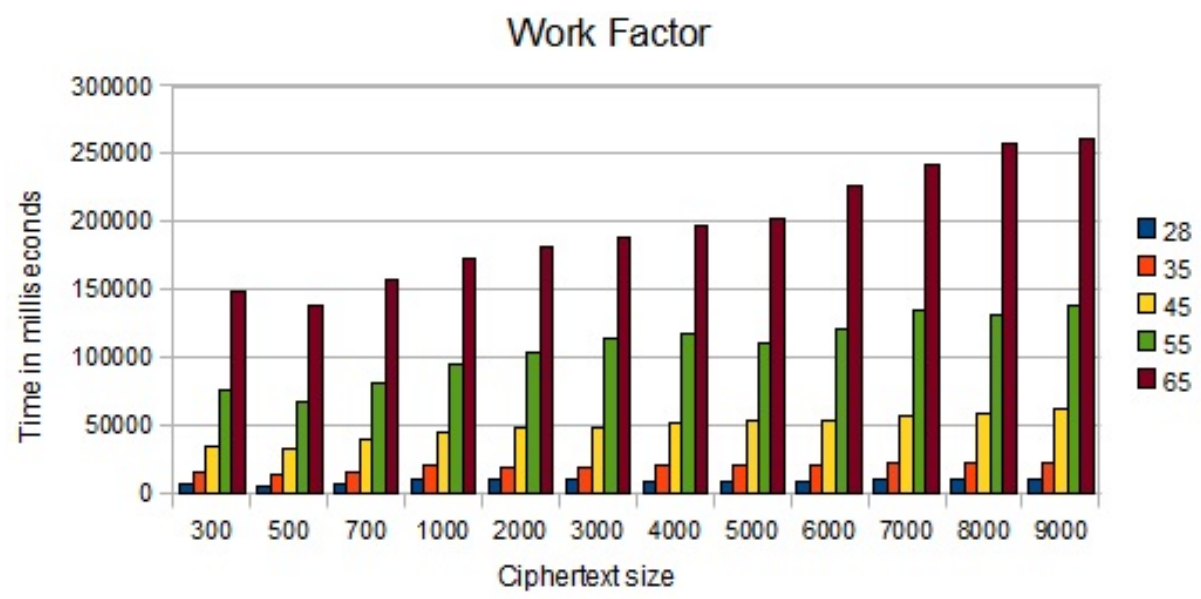

Figure 7: Program Timings

We tested a variety of simple and homophonic substitution ciphers. To test the accuracy of the implementation, we generated various ciphertexts and compared the attack results to the actual solutions. For each attack, we calculated the percentage of recovered plaintext symbols and plotted graphs to display our success rates.

The test cases discussed in this section cover different scenarios involving various ciphertext sizes, number of ciphertext symbols, and number of initial starting points provided to the inner hill climb layer. For all test cases, the plaintext is English and hence the $E$ matrix contains standard English digram statistics. Also, all tests were conducted twice, once using 27 plaintext symbols (English letters, plus space) and once using 26 plaintext symbols (English letters without space). The results were similar, so here we only include test cases using 27 plaintext symbols.

\subsection{Simple Substitution}

In this section, we briefly consider test cases where our algorithm is applied to a simple substitution cipher. For each test, we used our inner hill climb algorithm and the random initial key layer with $R=40$ initial starting points. Note that this algorithm is essentially equivalent to 40 iterations of Jakobsen's algorithm, with random initial starting points in each iteration. The plaintext consists of 27 symbols (26 letters and space), and the following ciphertext length were considered: 300,500 , $700,1000,2000,3000,4000,5000,6000,7000,8000$, and 10,000 characters. The graphs in Figure 8 present the results for these test cases.

From the graphs in Figure 8, we clearly see that as the length of the ciphertext increases, the score improves and the percentage of correct symbols increases. These results are as expected. 


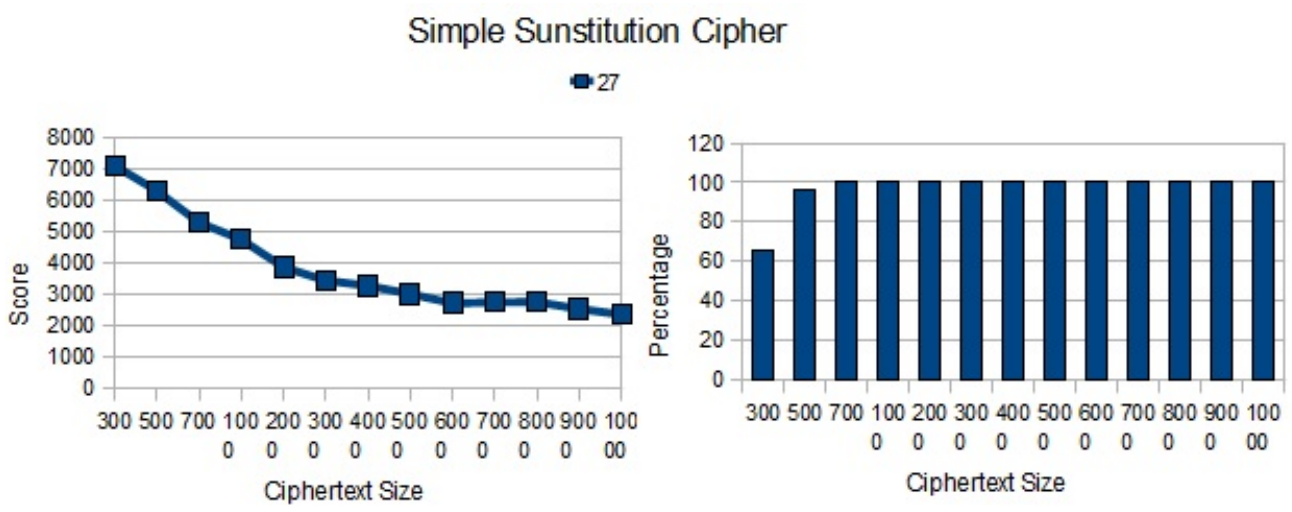

Figure 8: Simple Substitution Cipher

\subsection{Homophonic Substitution}

We applied our attack code to more than 1000 homophonic substitution test cases. For these test cases, ciphertext lengths ranged from 300 to 10,000 characters, cipher alphabet sizes ranged from 27 to 100, and the numbers of initial starting points ranged from 1 to 100. For all test cases reported here, the plaintext consists of 27 symbols (26 English letters plus the space).

In the remainder of this section, we summarize several test cases. These test cases only present a small subset of the tests that were conducted.

- Case 1: In this case, we test the inner hill climb layer. To do so, we assume that the outer hill climb generated the correct letter distribution, that is $n_{a}, n_{b}, \ldots, n_{z}$ exactly matches the actual key. Also, we use one initial random starting point.

- Number of ciphertext symbols: 27 to 63

- Ciphertext lengths: 300, 500, 700, 1000, 2000, 3000, 4000, 5000, 6000, 7000, 8000 , and 10,000

- Results: The graph in Figure 9 presents the final scores and the graph in Figure 10 presents the success rates. For clarity, a subset of the results from Figures 9 and 10 are presented in Figures 11 and 12, respectively. Note that with a success rate of about $80 \%$ or more, it is easy to manually recover the correct plaintext and key.

- Observations: As expected, the scores decrease (i.e., the solutions improve) as the size of the ciphertext increases, regardless of the number of ciphertext symbols. However, with limited ciphertext, the scores are poor.

- Case 2: This test case is the same as Case 1, except that we use 40 initial random starting points. That is, Case 1 tested the inner hill climb layer alone, while this case tests the inner hill climb and random initial key layers together. 
Homophonic Cipher Test Case 1

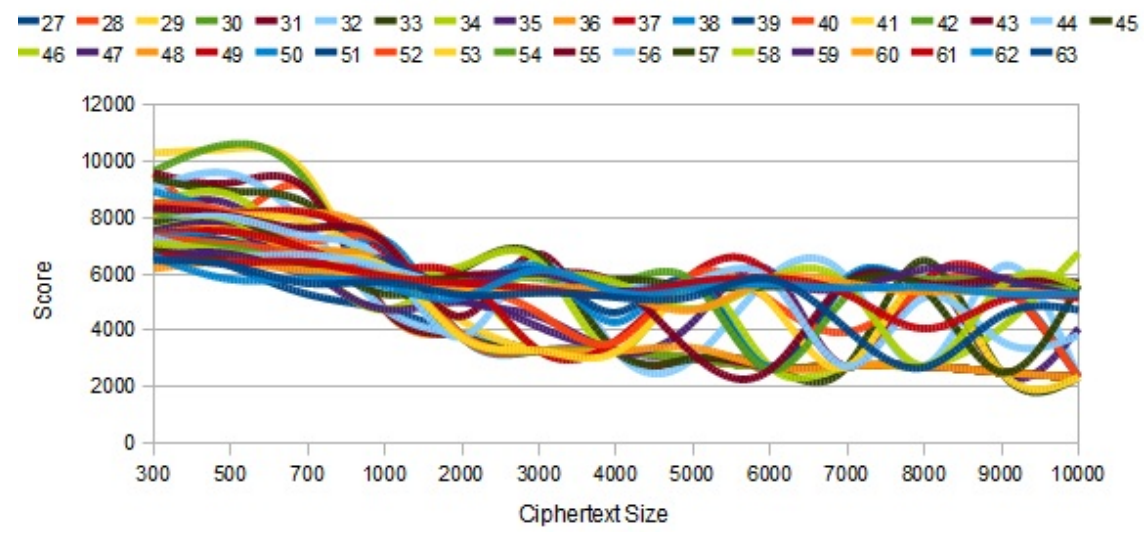

Figure 9: Homophonic Substitution: Case 1 Scores

Homophonic Cipher Test Case 1

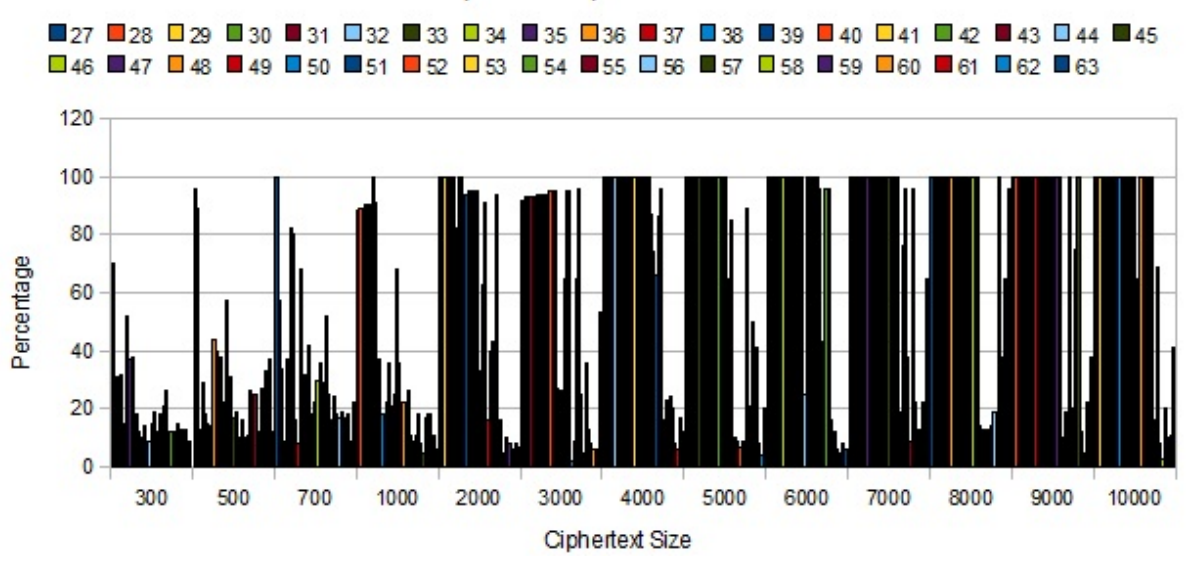

Figure 10: Homophonic Substitution: Case 1 Success Rate

- Number of ciphertext symbols: 27 to 63

- Ciphertext lengths: 300, 500, 700, 1000, 2000, 3000, 4000, 5000, 6000, 7000, 8000 , and 10,000

- Results: The graph in Figure 13 presents the final scores and the graph in Figure 14 presents the success rates. In Figures 15 and 16, we duplicate the results from Figures 13 and 14, and also include some instances with larger cipher symbol size.

- Observations: Including the random initial key layer greatly improved the results. It is interesting that for all cipher symbol sizes less than 100, the scores converge in a fairly similar fashion as the ciphertext size increases. 


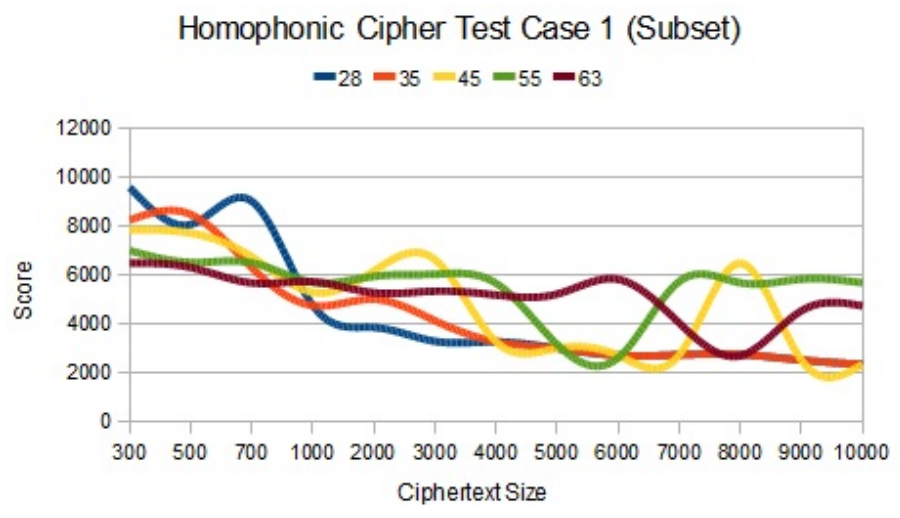

Figure 11: Homophonic Substitution: Case 1 (Subset) Scores Homophonic Cipher Test Case 1 (Subset)

$\square_{28} \square_{35} \square_{45} \square_{55} \square_{63}$

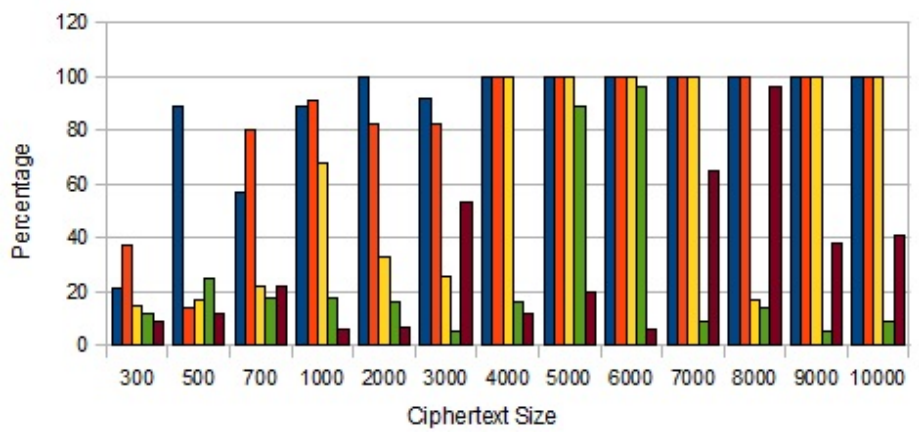

Figure 12: Homophonic Substitution: Case 1 (Subset) Success Rate

Also, from the graph is Figure 15, it appears that for homophonic substitutions with 100 or more cipher symbols, our results are likely to be poor, regardless of the size of the length of the ciphertext message.

- Case 3: Here, we duplicate much of the previous test case, but use 100 random starting points for the random initial key layer - as opposed to 40 in Case 2.

- Number of ciphertext symbols: 28, 35, 45, 55, and 63

- Ciphertext lengths: 300, 500, 700, and 1000

- Results: The graph in Figure 17 presents the final scores and the graph in Figure 18 presents the success rates.

- Observations: The results are not significantly improved over the previous test case, so we conclude that 40 initial starts is sufficient. 
Homophonic Cipher Test Case 2

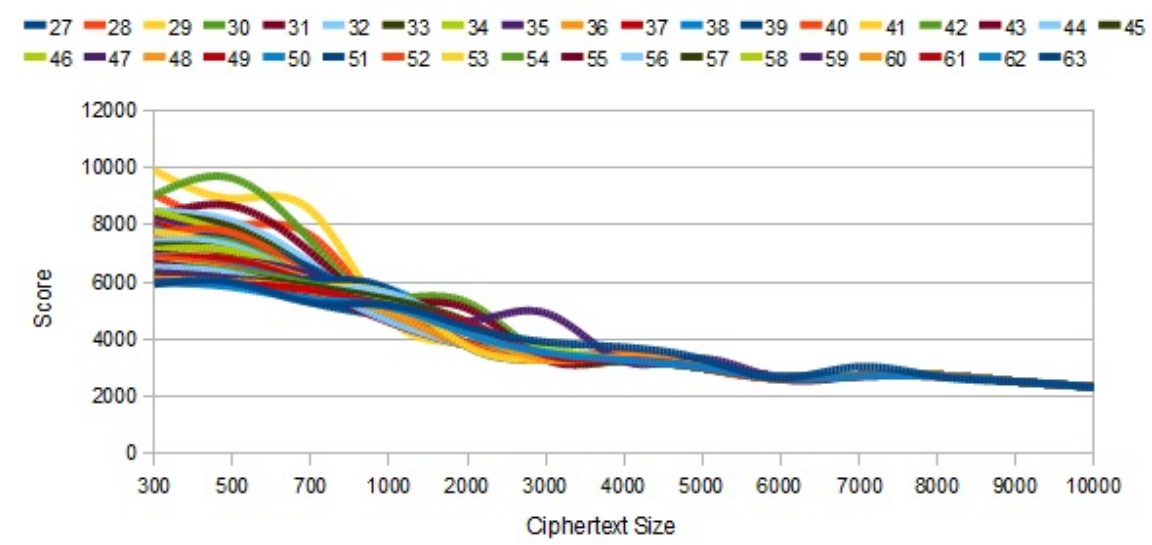

Figure 13: Homophonic Substitution: Case 2 Scores

Homophonic Cipher Test Case 2

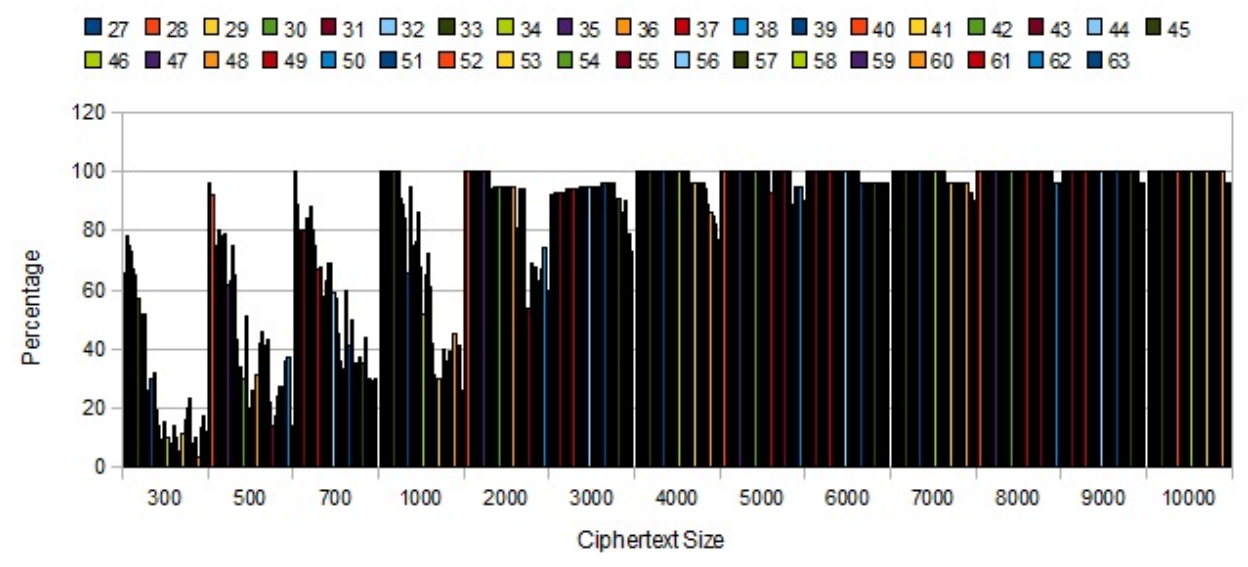

Figure 14: Homophonic Substitution: Case 2 Success Rate

- Case 4: Inner hill climb layer with random initial key layer (with 40 initial starting points per iteration) and outer hill climb layer. Note that this is the first test of our complete algorithm.

- Number of ciphertext symbols: 28, 35, 45, 55, and 63

- Ciphertext lengths: 300, 500, 700, 1000, 2000, 3000, 4000, 5000, 6000, 7000, 8000 , and 10,000

- Results: The graph in Figure 19 presents the final scores and the graph in Figure 20 presents the success rates.

- Observations: Including the outer hill climb layer gives us similar results to Case 2. This is a very positive finding since, in effect, Case 2 assumes the 


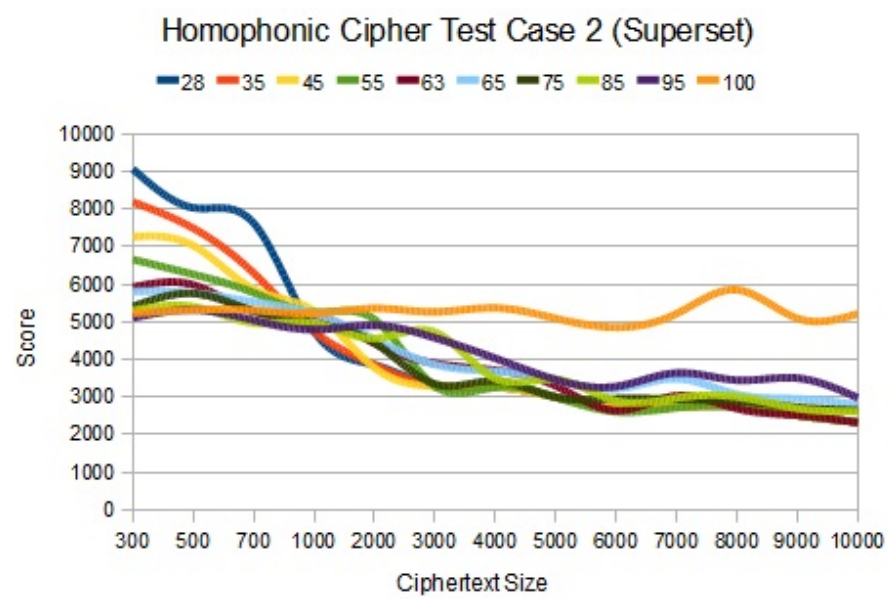

Figure 15: Homophonic Substitution: Case 2 (Superset) Scores

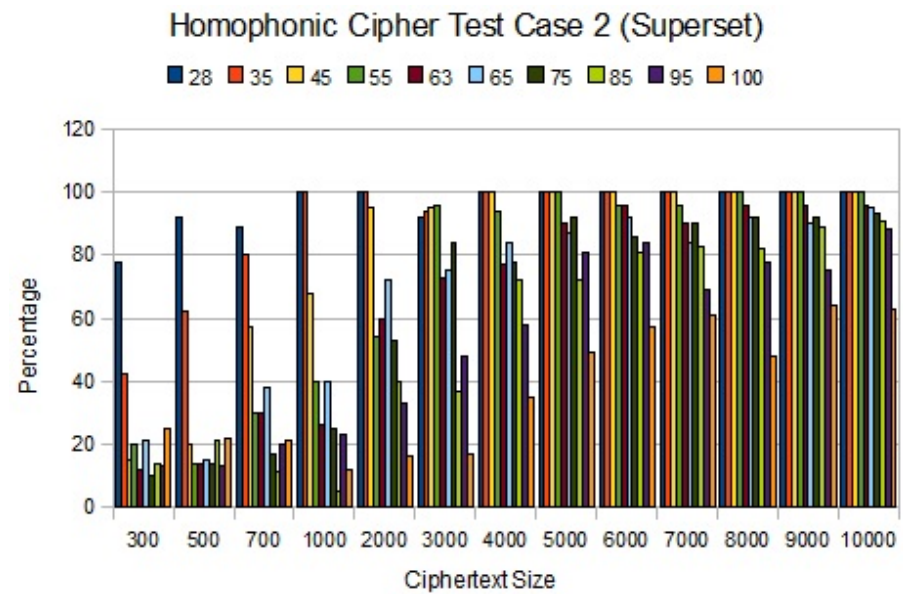

Figure 16: Homophonic Substitution: Case 2 (Superset) Success Rate

optimal output from the outer hill climb layer. The bottom line is that the results in this test case indicate that the outer hill climb layer is effective. Also, as with the previous test cases, the scores show a strong dependence on the ciphertext length.

Finally, we summarize the results for our homophonic substitution attack in the form of the 3-dimensional graph in Figure 21. The graph shows the success rate $(z$-axis) as a function of the ciphertext length ( $x$-axis), and number of ciphertext symbols ( $y$-axis). As noted above, a success rate of about $80 \%$ is sufficient to easily recover the plaintext 


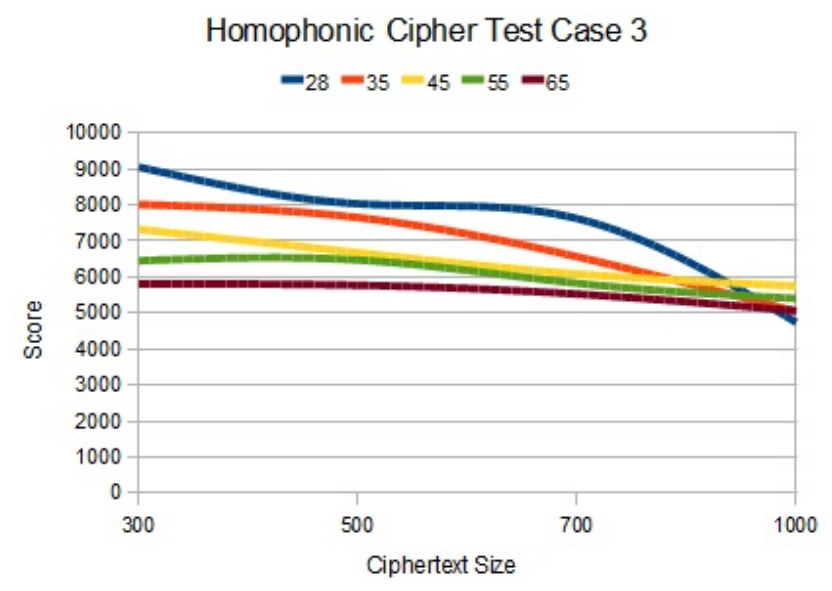

Figure 17: Homophonic Substitution: Case 3 Scores

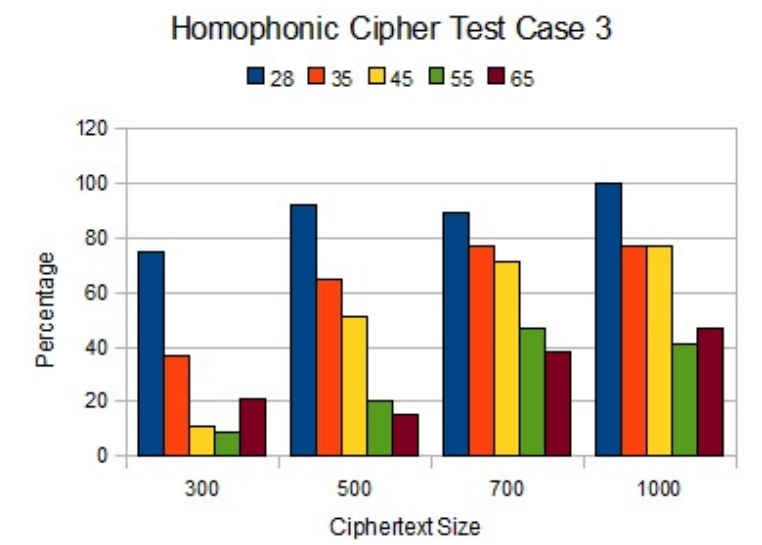

Figure 18: Homophonic Substitution: Case 3 Success Rate

\section{Zodiac Ciphers}

The Zodiac was a serial killer who claimed at least seven victims (five dead, two injured) in the San Francisco vicinity during the late 1960s and early 1970s [5]. Nobody was prosecuted for the Zodiac killings. During his killing spree, the Zodiac sent letters to local newspapers and police, often taunting police for their failure to determine his identity. Some of these letters included ciphers and apparent ciphers.

The "Zodiac 408" cipher - so-called because it has 408 ciphertext symbols-was a three-part ciphertext, with one part sent to the San Francisco Chronicle, one part sent to the San Francisco Examiner, and the third part sent to the Vallejo TimesHerald. The cipher, which is a homophonic substitution, was broken within six days of its publication by local school teachers, Donald and Bettye Harden [1]. 


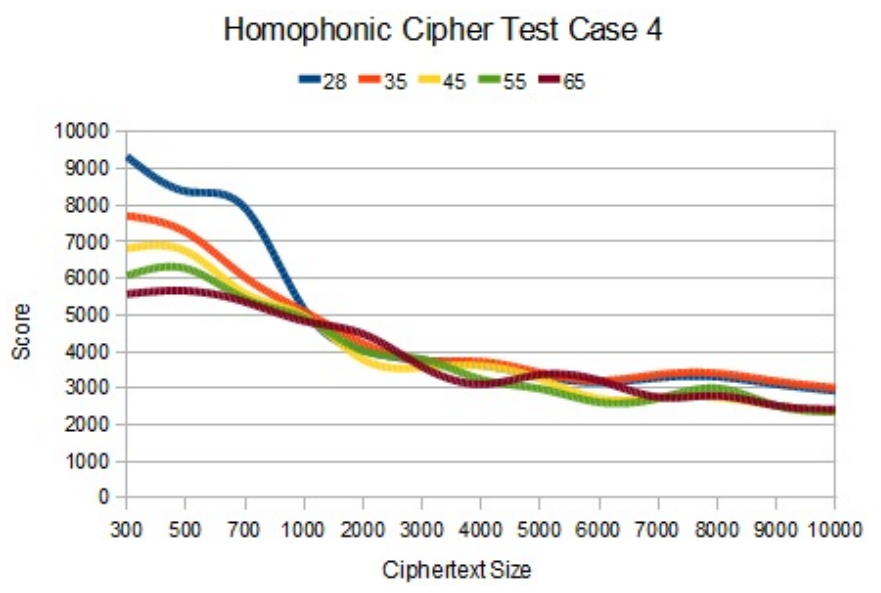

Figure 19: Homophonic Substitution: Case 4 Scores

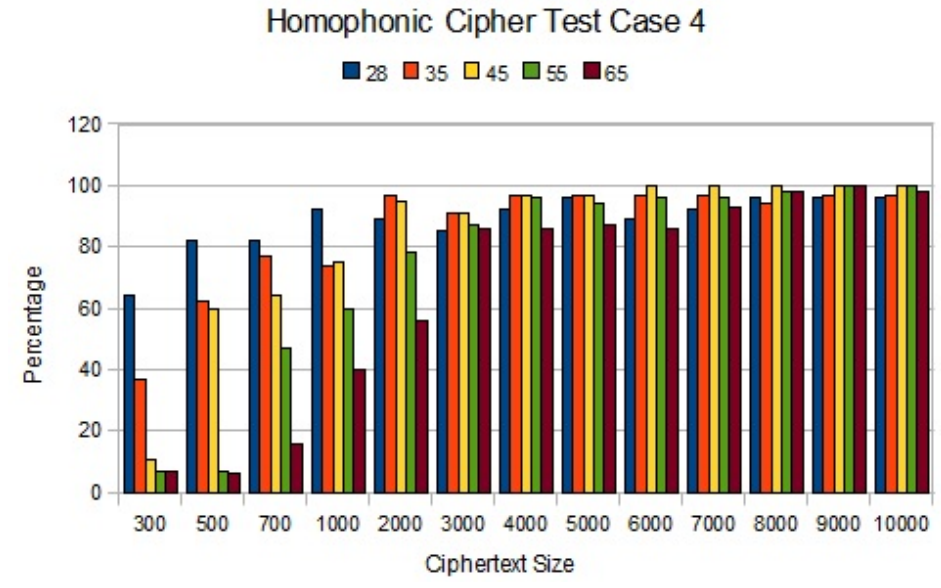

Figure 20: Homophonic Substitution Case 4: Success Rate

In addition to the Zodiac 408 cipher, the Zodiac killer sent three more messages that appear to be ciphertext. Two of these are very brief and therefore not amenable to cryptanalysis. However, the unsolved "Zodiac 340" (so-called because the ciphertext is of length 340) appears to offer some prospects for cryptanalysis.

Next, we discuss the solved Zodiac 408 cipher. Then we consider the Zodiac 340 and a related cipher challenge problem.

\subsection{Zodiac 408 Cipher}

The Zodiac 408 cipher is displayed in Figure 22. Note that each of the three parts of the ciphertext message is displayed as eight rows of 17 symbols. 


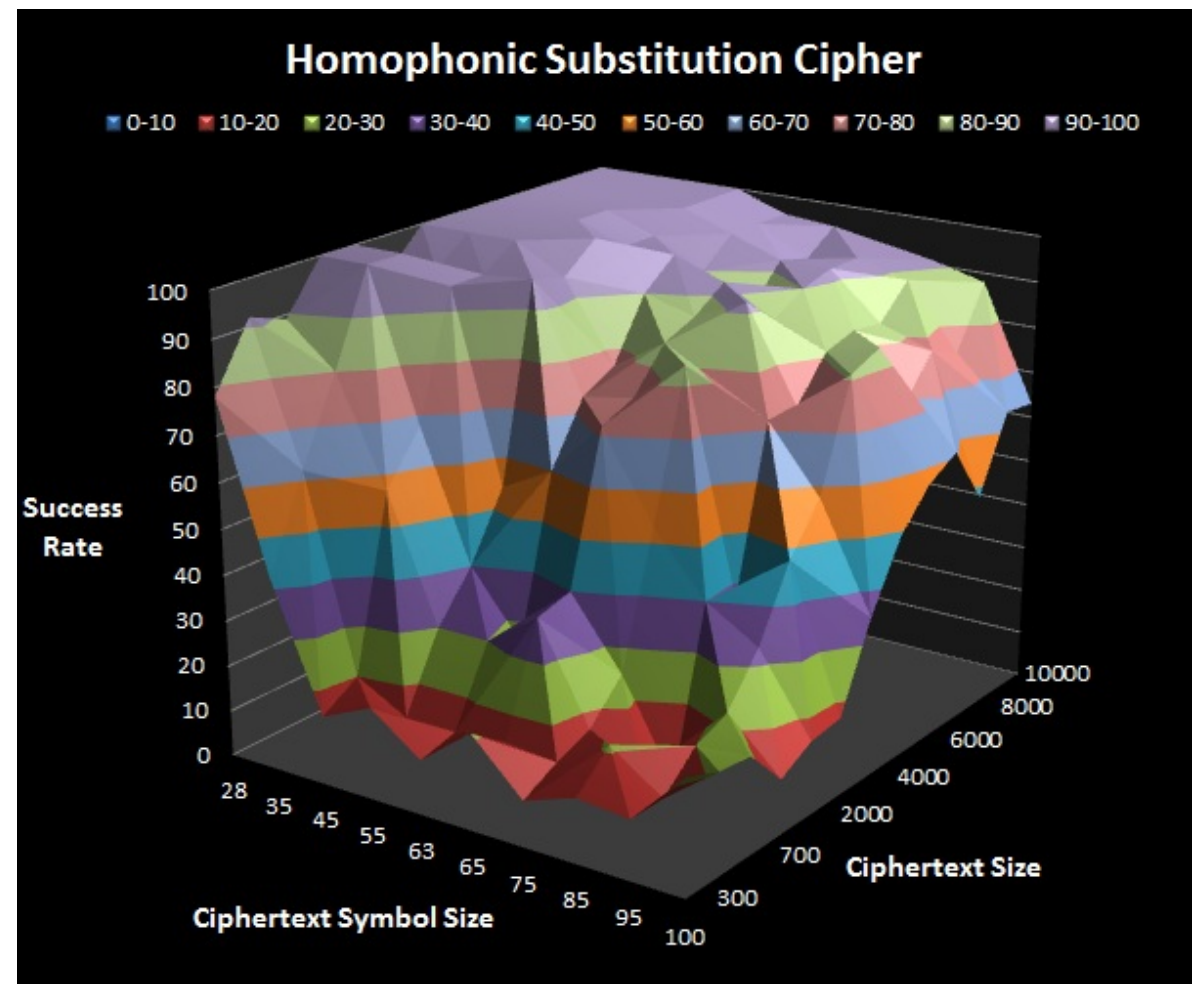

Figure 21: Homophonic Substitution Success Rates

The solution to the Zodiac 408 cipher is given in Table 11, where we display two rows of the original message per line, with a small gap between the two. Also, we have left a gap between each of the three parts.

Note that the misspellings appear in the original message. Some of these "misspellings" might be due to the fact that a thick-tipped pen was used, and several of the symbols are similar. Also, the final 18 symbols appear to be filler used to make the cipher fit a predetermined grid [6].

The frequency distribution for the Zodiac 408 plaintext is given in Table 12. The letters $\mathrm{J}, \mathrm{Q}$, and $\mathrm{Z}$ do not appear in the plaintext.

\subsection{Zodiac 340 Cipher}

The unsolved Zodiac 340, which appears in Figure 23, is reminiscent of the Zodiac 408. This apparent ciphertext was mailed to the San Francisco Chronicle on November 8, 1969 [16]. There have been many proposed solutions to the Zodiac 340, but none of the "solutions" published to date holds up under scrutiny. For recent examples of proposed solutions, see [18] or [22]. The website [14] gives a good analysis of the flaws in the supposed solution [18]. 
1. TIMES HERALD $8 / 1 / 69$

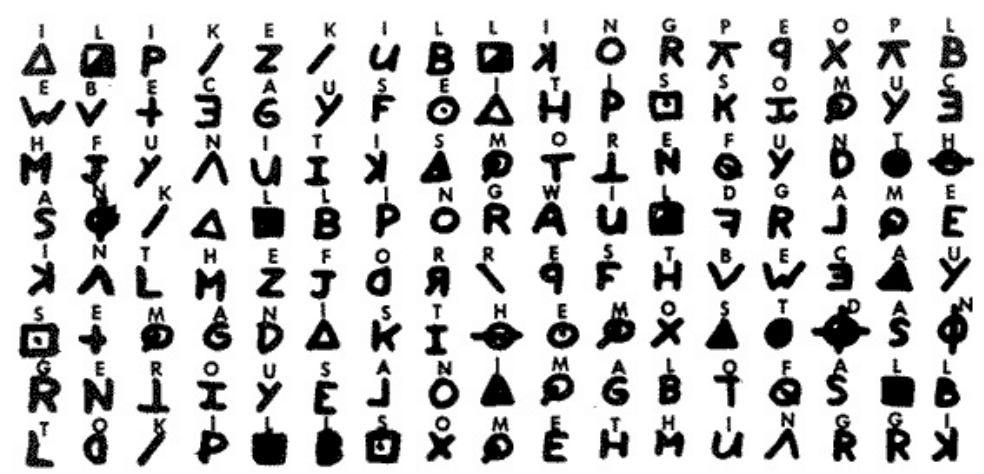

2. CHRONICLE $8 / 1 / 69$

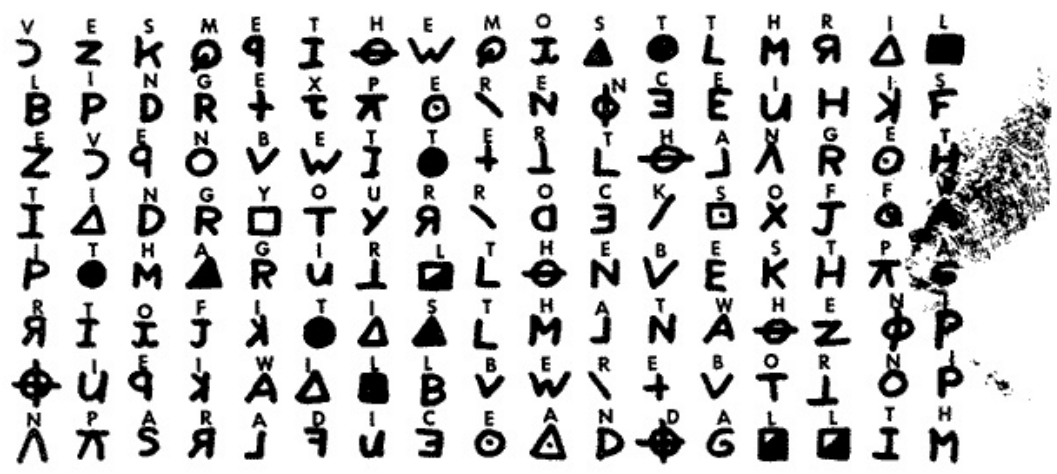

3. EXAMINER $8 / 1 / 69$

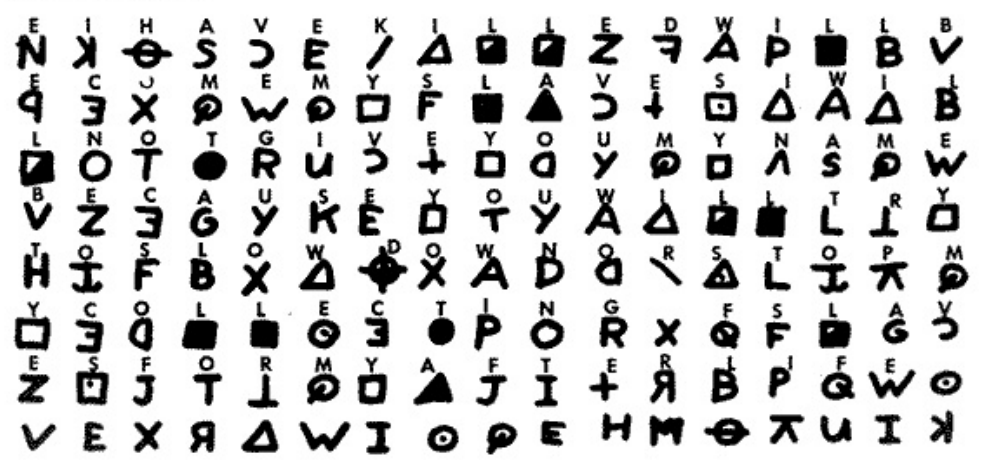

Figure 22: Zodiac 408 Cipher [19]

It is possible that the Zodiac 340 is a homophonic substitution combined with another encryption technique. Of course, another possibility is that the Zodiac 340 is not a ciphertext - it could be nothing more than a random collection of symbols. For the remainder of this paper, we assume the Zodiac 340 is a homophonic substitution. 
Table 11: Zodiac 408 Plaintext

ILIKEKILLINGPEOPL EBECAUSEITISSOMUC HFUNITISMOREFUNTH ANKILLINGWILDGAME INTHEFORRESTBECAU SEMANISTHEMOSTDAN GEROUSANAMALOFALL TOKILLSOMETHINGGI

VESMETHEMOSTTHRIL LINGEXPERENCEITIS EVENBETTERTHANGET TINGYOURROCKSOFFW ITHAGIRLTHEBESTPA RTOFITISTHAEWHENI DIEIWILLBEREBORNI NPARADICEANDALLTH

EIHAVEKILLEDWILLB ECOMEMYSLAVESIWIL LNOTGIVEYOUMYNAME BECAUSEYOUWILLTRY TOSLOIDOWNORSTOPM YCOLLECTINGOFSLAV ESFORMYAFTERLIFEE BEOROETEMETHHPITI

Table 12: Zodiac 408 Plaintext Frequency Counts

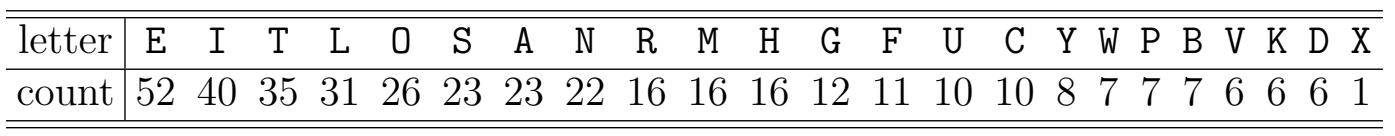

We conducted several tests on the Zodiac 340 cipher. Two typical test cases are summarized below.

- Case 1: Inner hill climb with 40 random starts (no outer hill climb)

- Plaintext symbols: 26 letters (no space)

- Score: 6092

- Putative plaintext:

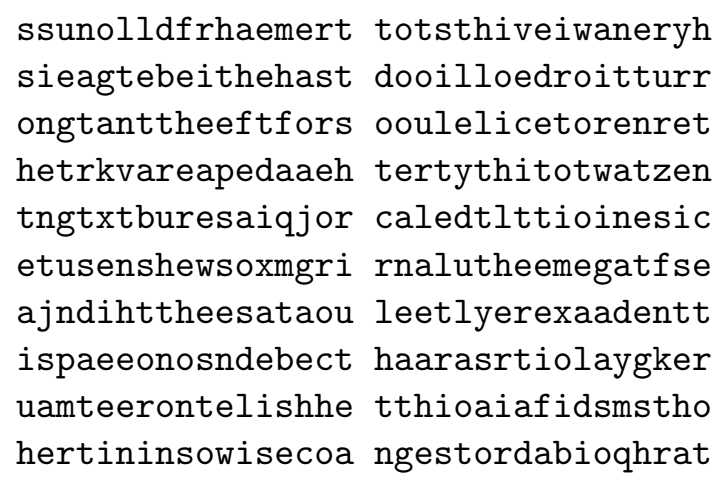




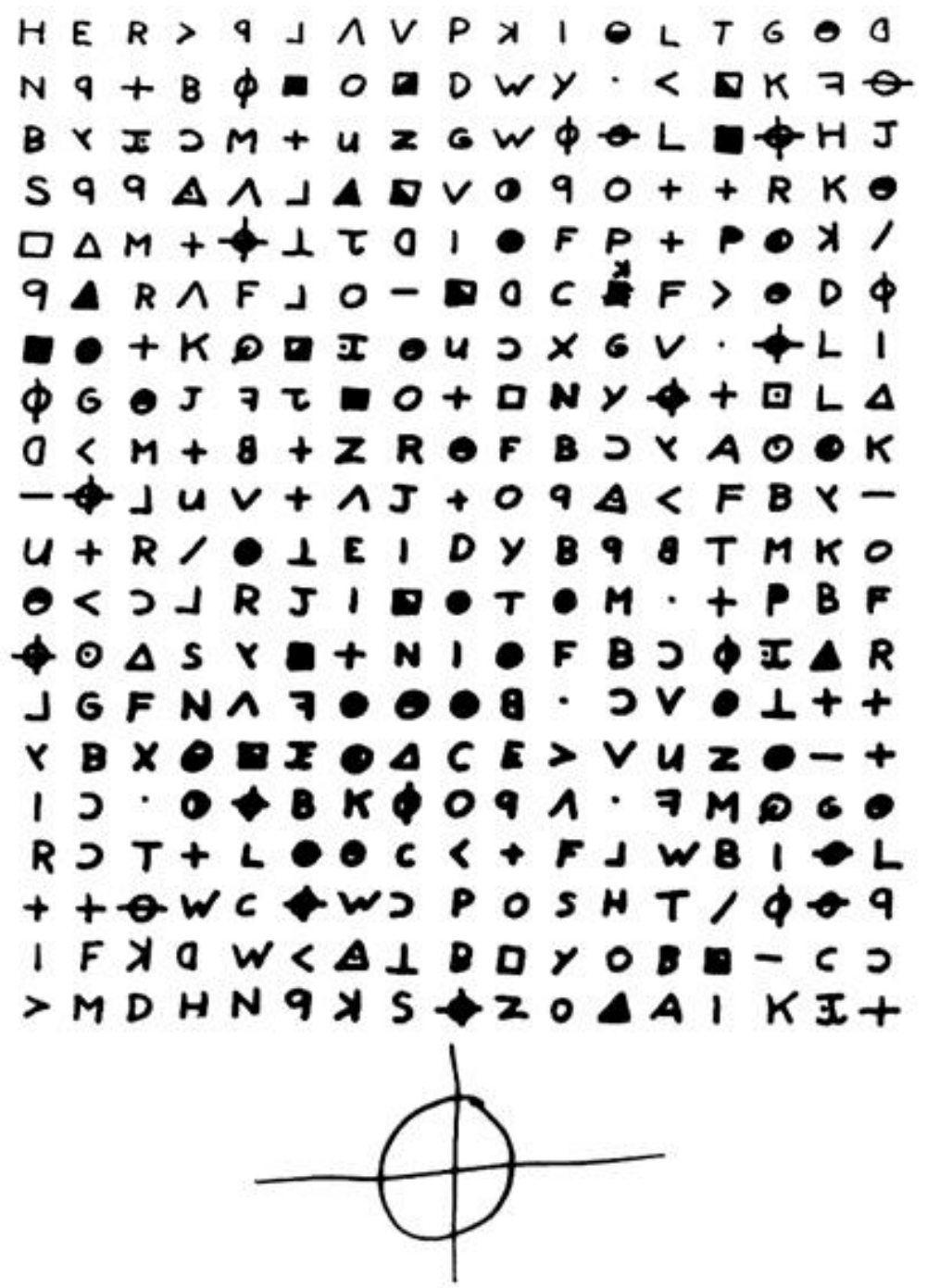

Figure 23: Zodiac 340 Cipher [16]

- Case 2: Outer hill climb with inner hill climb and 40 random starts

- Plaintext symbols: 26 letters (no space)

- Score: 5868

- Putative plaintext

passturinytistref otinatlkealdethen

ngidwineraanstmpb atthruetiqtliishe

cowimerfthonintys tesrouletfryoseea

thihakvendoridmst arebertlicolmizso 


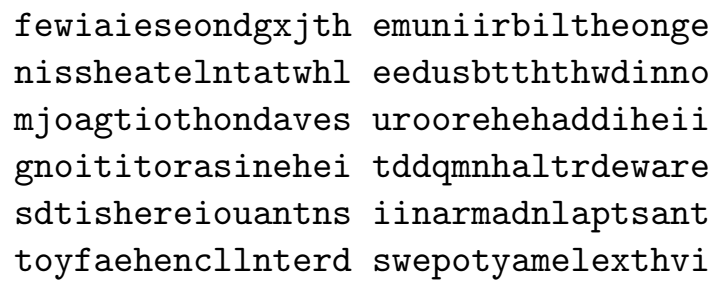

Obviously, neither of these solutions is particularly impressive. Also, neither score is indicative of a strong match to English.

\subsection{MysterTwister Zodiac Challenge}

The MysteryTwister website [11] includes a large number of interesting cipher challenges, ranging from pencil-and-paper to research-level problems. One of the challenges posted on the MysterTwister website was inspired by the Zodiac cipher. We applied our homophonic substitution attack on this challenge problem. Note that the challenge problem ciphertext has 340 character, with 65 distinct symbols. That is, the challenge was designed to mimic the Zodiac 340 cipher.

We consider two cases. First, we use English digrams in the target matrix $E$. That is, we follow the same procedure as used in all of the examples presented to this point. Second, we consider a case with the Zodiac 408 plaintext as the expected "language," that is, we replace the $E$ matrix based on English, with the digram statistics of the Zodiac 408 plaintext. Since the Zodiac 408 is known to be the Zodiac's writing, this might better model the underlying plaintext than standard English. The results for these two test cases are summarized below.

\section{- Case 1}

- Outer hill climb layer with initial random key layer (40 initial starts per iteration) and inner hill climb layer

- E matrix: English digrams

- Score: 5567

- Putative plaintext:

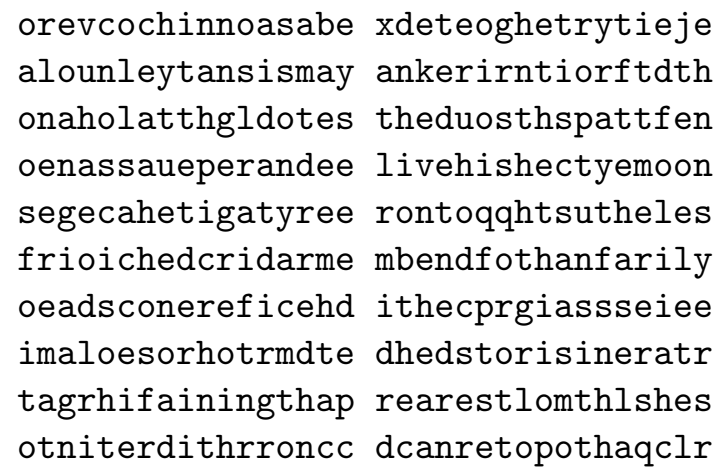




\section{- Case 2}

- Outer hill climb layer with initial random key layer (40 initial starts per iteration) and inner hill climb layer

- E matrix: Zodiac 408 plaintext digrams

- Score: 4229

- Putative plaintext:

ilidetillingreopl dbecaeseittisomal
ezenitiimofeaunth anjilltngwildgame
inthezorrestbecau semanisthenostdan
gefousaninalosall todillsomethinggi
vesmethemostthril lingexqerenceitis
dteiwillbereborni npafadiceandallth
eieavetilledwillb ecomenrslavesiwil
lnotgiveroemrname belauserouwilltrr
toslowdownofstorn rlollectingozslav
essormraaterlisee beoftetenetheqitt

From the putative plaintext obtained in Case 2, we can complete the solution manually. The solution is given by (with spaces added):

I like killing people because it is so much fun it is more fun than killing wild game in the forrest because man is the most dangerous animal of all to kill something gives me the most thrilling experence it is die I will be reborn in paradice and all the I have killed will become my slaves I will not give you my name because you will try to slow down or stop my collecting of slaves for my afterlife ebeorietemethhpiti

As an additional experiment, we modified the outer hill climb so that it does a more thorough, but slower, hill climb. In this modified hill climb, we "swap" all adjacent pairs of elements, and then iterate, that is, we again swap all adjacent pairs of elements, and again, and so on. This continues until we complete one entire iteration without any swap improving the score. We refer to this as the "slow" outer hill climb, as opposed to our standard outer hill climb which we call the "fast" approach. Our test results comparing the slow versus fast outer hill climb are given in Table 13 .

From Table 13 we again see that using the Zodiac 408 statistics for the $E$ matrix gives much better results than standard English. However, it is interesting to note that the slow hill climb gives significantly better results when the Zodiac 408 statistics are used, while it actually yields worse results when English statistics are used. The explanation appears to be that the slow hill climb does indeed yield "better" results, in the sense of producing putative plaintext that more closely models the target matrix $E$. However, the Zodiac challenge cipher does not fit the standard English model particularly well. Consequently, as the hill climb better matches the English matrix $E$, it match the true plaintext less. In contrast, using the Zodiac 408 statistics 
Table 13: MysteryTwister Zodiac Challenge

\begin{tabular}{c|lc}
\hline \hline Case & \multicolumn{1}{|c}{ Description } & Percentage \\
\hline $1 \mathrm{~F}$ & Fast outer hill climb and English stats & $13.00 \%$ \\
$1 \mathrm{~S}$ & Slow outer hill climb and English stats & $4.00 \%$ \\
$2 \mathrm{~F}$ & Fast outer hill climb and Zodiac 408 stats & $70.00 \%$ \\
$2 \mathrm{~S}$ & Slow outer hill climb and Zodiac 408 stats & $84.00 \%$ \\
\hline \hline
\end{tabular}

(which are virtually identical with those of the Zodiac challenge), a stronger match to the $E$ matrix, as in Case $2 \mathrm{~S}$ of Table 13, produces more accurate results.

The results in this section strongly indicate that it should be worthwhile to consider specialized "language" models when trying to break the Zodiac 340. It might also be useful to employ the slow outer hill climb, but that appears to yield a more modest improvement. However, for the Zodiac 340, we have a brief ciphertext (only 340 symbols) and a relatively large alphabet (62 symbols). From Figure 21, we see that for these parameter values, we will need every possible advantage to have a reasonable chance of breaking the cipher.

\section{Conclusions and Future Work}

We designed and implemented an efficient attack on homophonic substitution ciphers. The attack utilizes a nested hill climb approach, and can be viewed as a generalization of the fastest known attack on simple substitution ciphers. The algorithm was implemented and extensively tested. We were able to recover at least $80 \%$ of the plaintext characters for ciphers having 42 ciphertext symbols or less, provided we have a ciphertext of 1000 or more characters. If the ciphertext length is at least 3000 characters, then we achieve the same level of success for ciphers with 75 or fewer ciphertext symbols.

These results indicate that the Zodiac 340 cipher-assuming it is a homophonic substitution - may be out of range for our attack. However, tests indicate that with a more accurate plaintext "language" model and a more thorough outer hill climb, we should have a realistic chance of breaking a cipher such as the Zodiac 340. Therefore, (again, assuming the Zodiac 340 is actually a homophonic substitution), our best chance of success is, perhaps, to devise a strong language model for the unknown plaintext. This would require some degree of insight and luck.

There are several possible improvements to our attack algorithm. The slow outer hill climb that was briefly discussed in Section 6.3 is one such improvement. Another possible modification to the outer hill climb is to make multiple "swaps" with a given pair until the score no longer improves, before moving on to the next pair. Perhaps a combination of these two modifications to the outer hill climb layer would 
be optimal, with respect to improving the score. However, these modifications would also significantly increase the cost of the algorithm.

It might seem natural to try to extend the scoring to include, say, trigrams. However, swapping and scoring would both become extremely complex, and the work factor would increase exponentially. Dictionary-based attacks also seem tempting, but have similar drawbacks.

A potentially profitable modification to our algorithm would be to include a "crib" feature. That is, the user could specify a word that might appear in the plaintext, and this word could be tried at every offset in the ciphertext. This would be a straightforward modification of the algorithm.

Various heuristic search techniques could be combined with the general approach discussed in this paper. For example, the outer hill climb could be replaced by a genetic algorithm [8]. While genetic algorithms have been applied to homophonic substitution ciphers [3, 13], they have not been used in a manner similar to the method suggested here. Other heuristic search techniques could also be considered.

\section{References}

[1] A 'murder code' broken, San Francisco Chronicle, August 9, 1969, http://www.flickr.com/photos/seanutbutter/2462841231/

[2] Announcing the Advanced Encryption Standard (AES), NIST, FIPS 197, November 26, 2001, http://csrc.nist.gov/publications/fips/fips197/fips-197.pdf

[3] P. Basavaraju, Heuristic-search cryptanalysis of the Zodiac 340 cipher, Master's report, Department of Computer Science, San Jose State University, 2009, http://www.cs.sjsu.edu/faculty/stamp/students/ kanagalakatte_pallavi.pdf

[4] K. Briggs, English and Latin digram and trigram frequencies, http://keithbriggs.info/documents/english_latin.pdf

[5] G. Claston, 340-cipher - overview and examination, http://www .zodiackiller.com/mba/zc/69.html

[6] Glurk, The final 18 symbols are nothing but filler!, http: //www . zodiackillerfacts. com/forum/viewtopic $\cdot$ php? $f=49 \& t=423$

[7] T. Jakobsen, A fast method for the cryptanalysis of substitution ciphers, Cryptologia, Vol. 19, pp. 265-274, 1995

[8] D. Kreher and D. Stinson, Combinatorial Algorithms: Generation, Enumeration and Search, CRC Press, 1998

[9] Local Obstacle Avoidance and hill-climbing, GamePlayDev, http://www.gameplaydev.com/2010/08/ local-obstacle-avoidance-and-hill-climbing/ 
[10] J. Mathai, History of computer cryptography and secrecy system, http://www.dsm. fordham.edu/ mathai/crypto.html

[11] MysteryTwister C3: The crypto challenge contest, http://www . mysterytwisterc3.org/

[12] E. Olson, Robust dictionary attack on short simple substitution ciphers, Cryptologia, Vol. 31, No. 4, pp. 332-342, 2007, http://april.eecs.umich.edu/pdfs/olson2007crypt.pdf

[13] D. Oranckchak, Evolutionary algorithm for decryption of monoalphabetic homophonic ciphers encoded as constraint satisfaction problems, GECCO '08, July 12-16, 2008, http://oranchak.com/t14pap379-oranchak.pdf

[14] D. Oranckchak, Corey Starliper claims to have solved the 340-character Zodiac Killer cipher, http://oranchak.com/zodiac/corey/hoax.html

[15] P. C. Pop and I. Zelina, Heuristic algorithms for generalized minimum spanning tree problem, Proceedings of ICTAMI 2004, http://www .uab.ro/auajournal/acta8/Pop-Zelina.pdf

[16] Preliminary report on project MK-ZODIAC, 2011, http://mk-zodiac.com/game.html

[17] ROT 13 - simple substitution cipher, http://www.tech-faq.com/rot-13.html

[18] B. Schillemat, Has the code of the Zodiac killer been cracked?, Forest City Patch, http://fostercity.patch.com/articles/ has-the-code-of-the-zodiac-killer-been-cracked

[19] Solved Zodiac 408 cipher, http://wiki.zodiac-ciphers.dreamhosters.com/ wiki/Solved_408-character_cipher

[20] M. Stamp and R. M. Low, Applied Cryptanalysis: Breaking Ciphers in the Real World, Wiley, 2006

[21] M. Stamp, Information Security: Principles and Practice, second edition, Wiley, 2011

[22] The Zodiac 340 cipher solved, http://www . opordanalytical.com/articles1/zodiac-340.htm 